\title{
Studies and Measurements of Irradiated Solid Polarized Target Materials
}

\author{
Jonathan E. Mellor \\ Vienna, Virginia
}

B.S., The College of William and Mary, 2001

A Thesis Presented to the Graduate

Faculty of the University of Virginia

in Candidacy for the Degree of Master of Science

Department of Physics

University of Virginia

August 2006

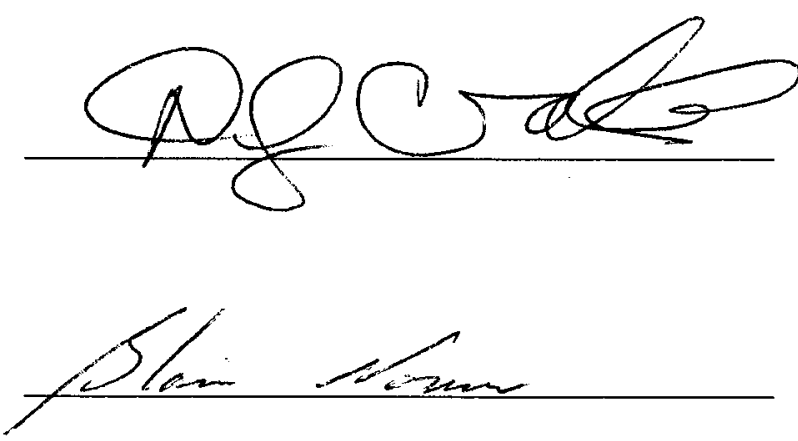




\begin{abstract}
Experiments carried out at Thomas Jefferson National Accelerator Facility (JLab) in Newport News, Virginia, and other national labs, require the use of solid polarized targets that are used to study the internal structure of either the proton or neutron. The most common target materials used in such experiments include ammonia, butanol, pentanol, lithium hydride and their deuterated versions.

A technique called Dynamic Nuclear Polarization (DNP) is used to polarize these materials at low temperatures and high magnetic fields. This method requires the introduction of paramagnetic radicals. These radicals are most commonly introduced either chemically or through irradiation. Historically, irradiations have been done at several different national laboratories with varying parameters at each laboratory. These parameters: incident electron energy, irradiation dewar geometry and cryogenic liquid, can have a significant effect on the dose deposited into the material. This thesis uses EGS4, a Monte Carlo simulation tool, to simulate and normalize irradiations done at the National Institute for Science and Technology's Medical and Industrial Radiation Facility. The results of these investigations, which are consistent with experimental polarization measurements, indicate that the above parameters have a significant effect on the dose deposited.
\end{abstract}

A secondary aim of this thesis is to simulate the target beam heating for experiments carried out at the Stanford Linear Accelerator and the High Intensity Gamma-Ray Source at Duke University. Such predictions are necessary to estimate the cooling power necessary to overcome the target heating due to the presence of the beam.

Lastly, the results of a series of polarization measurements are given. These measurements indicate that d-butanol, irradiated with a dose of $2-3 \times 10^{15}$ electrons $/ \mathrm{cm}^{2}$, can achieve a polarization of up to $+62.5 \%$. 


\section{Contents}

1 Introduction $\quad 6$

2 Solid Polarized Targets 11

2.1 Thermal Equilibrium Polarization . . . . . . . . . . . . . . . . . 11

2.2 Dynamic Nuclear Polarization . . . . . . . . . . . . . . . . . . . . . 14

2.3 Nuclear Magnetic Resonance . . . . . . . . . . . . . . . . . . . . . 17

2.4 Cryostat, Refrigerator and Magnet . . . . . . . . . . . . 19

2.5 Polarization Analysis . . . . . . . . . . . . . . . . . . . . 21

2.6 Polarization Error . . . . . . . . . . . . . . . . . . 27

2.7 Target Materials . . . . . . . . . . . . . . . . . . . . . . . . . . . . . . . . . . . . 28

2.8 Target Material Preparation . . . . . . . . . . . . . . . . . 29

2.9 Target Irradiations . . . . . . . . . . . . . . . . . . . . . . . . . . . . 33

$\begin{array}{lll}3 & \text { EGS4 } & 37\end{array}$

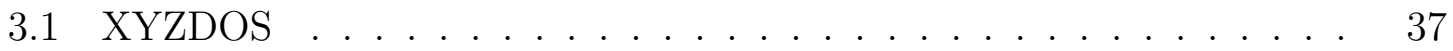

3.2 The Radiation of Transport-Shower Processes . . . . . . . . . . . . . 39

$3.3 \mathrm{NH}_{3}$ Irradiation . . . . . . . . . . . . . . . . . . . . . . . . . 40

3.4 Duke University Laboratory . . . . . . . . . . . . . . . . . . . . . . . . . 45

3.5 Stanford Linear Accelerator . . . . . . . . . . . . . . . . . . 47

4 Polarization Measurements $\quad 56$

4.1 Deuterons . . . . . . . . . . . . . . . . . . . 57

4.2 Ammonia . . . . . . . . . . . . . . . . . . 59

4.3 Other Protonated Materials . . . . . . . . . . . . . . 60

4.4 Conclusions/Outlook . . . . . . . . . . . . . 61

A Instructions for running EGS4 65

B Record information for input.inp $\quad 69$

$\begin{array}{lr}\mathrm{C} \text { input.inp } & 72\end{array}$ 
CONTENTS

D pegs4.inp

E Sample EGS4 Output 


\section{List of Figures}

2.1 Energy level diagram for an electron-proton system in a magnetic field. Electron-proton systems driven from $|B\rangle$ to $|C\rangle$ with microwaves of frequency $\left(\mu_{e}-\mu_{n}\right) B / h$ will quickly decay back down to $|A\rangle$. Likewise systems irradiated with frequency $\left(\mu_{e}+\mu_{n}\right) B / h$ will be driven from $|A\rangle$ to $|D\rangle$ from where they will quickly decay to $|B\rangle \ldots \ldots \ldots$

2.2 The Liverpool Q-Meter. Adapted from [14] . . . . . . . . . . . 20

2.3 The insert stick. Not to scale. Adapted from: $[13] \ldots \ldots . . . . .22$

2.4 The SLAC Oxford Cryostat which can, in conjunction with the $4 \mathrm{He}$ refrigerator cool target materials to $\sim 1 \mathrm{~K}$. The $\mathrm{LN}_{2}$ shield on the outside acts as the outer heat shield. The magnet dewar keeps the magnet at $4 \mathrm{~K}$. Liquid is passed from this region to the refrigerator by means of a transfer tube (not shown). . . . . . . . . . . . .

2.5 The raw signal is shown in the upper left hand corner. First, the baseline is subtracted from that signal giving the 'Subtracted Signal'. A polynomial is fitted to the wings of it. That polynomial is then subtracted from the 'Subtracted Signal' giving the 'Polysubtracted Signal'. The resulting signal is then integrated. The polarization is directly proportional to the area underneath the 'Polysubtracted Signal'. The constant of proportionality is the called the calibration constant and the polarization can be calculated using Equation 2.17. Figure adapted

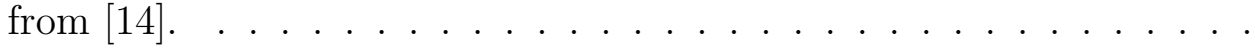

2.6 A typical deuteron signal. Note the characteristic double peaks associated with its quadropole moment. . . . . . . . . . . . .

2.7 The newly-modified ammonia freezing apparatus. It is now constructed entirely out of stainless steel to resist corrosion. After the system is flushed with $\mathrm{N}_{2}$ and pumped down with the mechanical pump, ammonia gas is cryopumped from the gas bottle on the left to the ammonia freezing tube, which is immersed in a bath of liquid nitrogen. . . . . . 
2.8 Newly designed ammonia freezing tube. The tube is made with stainless steel to be corrosion resistant. The end-cap is secured by eight screws and a indium seal to assure that it is leak-tight at low temperatures yet is easily unscrewed to allow the froozen slug to be removed.

2.9 The material is held in the irradiation basket, which is immersed in liquid argon. The outer vacuum is held a high vacuum with a diffusion pump. Electrons, incident from the left have to travel through a total of $0.71 \mathrm{~cm}$ of aluminum and several centimeters of liquid argon before reaching the material. Figure is not to scale and dimensions are approximate. . . . . . . . . . . . . .

3.1 "New", "Middle" and "Old" basket designs and their positions relative to the dewar wall. The "New" and "Middle" baskets are similar in position, although the Middle one is slightly longer. The "Old" basket is pushed much further forward than the newer models. All are 2.54 $\mathrm{cm}$ in diameter.

3.2 Total integrated energy deposited as a function of $\mathrm{Z}$. Green $=\mathrm{Al}+\mathrm{Ar}$, Pink $=\mathrm{Al}+\mathrm{NH}_{3}$, Light Blue $=$ Ar, Dark Blue $=\mathrm{Ar}+\mathrm{NH}_{3}$, Red $=$ $\mathrm{NH}_{3}$. All curves have approximately the same shape, but those configurations with an initial aluminum wall have more energy deposited. The other materials make little difference in the amount of energy deposited. 43

$3.3 \mathrm{dE} / \mathrm{dz}$ as a function of Z. Nearly all of of the dose is deposited within the first $6 \mathrm{~cm}$ of the target length. . . . . . . . . . . . . .

3.4 Intensity of a collimated coherent bremsstrahlung at four different energy levels. The resulting photon beam has a sharp peak around a single energy, but is non-zero are higher and lower energies. Adapted from: $[22] \ldots \ldots \ldots \ldots \ldots \ldots$

3.5 The deposited energy per photon as a function of the incident energy has a similar shape for both case of no magnetic field and a $6.5 \mathrm{~T}$ magnetic field. However, in the case of the $6.5 \mathrm{~T}$ magnetic field, the deposited energy is slightly less for low energies. . . . . . . . . .

3.6 The energy deposited as function of incident energy is much less for the Transverse $(\hat{y})$ direction than it is for the Longitudinal $(\hat{z})$ direction for energies less than approximately $5 \mathrm{GeV}$. . . . . . . . . .

3.7 Two cases: one with a $0.5 \mathrm{~cm}$ area and one with a $0 \mathrm{~cm}$ incident area are shown to demonstrate the effects of spot size on the deposited energy. The size has little effect on the deposited energy per photon. As a function of $\mathrm{Z}$ the energy deposited is nearly linear for the 450 $\mathrm{MeV}$ and $45 \mathrm{GeV}$ cases. It is only with the $4.5 \mathrm{MeV}$ case that the energy deposited decreases as the photons shower progresses through the material. . . . . . . . . . . . . . . . . . . . 
3.9 Dose deposited as a function of $\mathrm{x}$ and $\mathrm{y}$ in arbitrary units. From this plot it is easily seen the most of the dose is deposited in the rastered

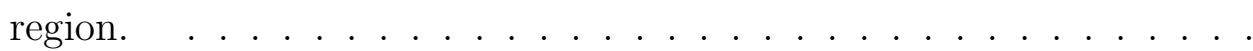

3.10 Photon flux with LPM effect. The $1 / \mathrm{k}$ spectrum produced by the bremsstrahlung effect is reduced by a factor of 25 for photons below $40 \mathrm{MeV}$. The photon flux is normalized to $10^{12}$ photons/second. . . .

3.11 Total power deposited by the SLAC photon beam with the LPM effect included. Most of the deposited power is due to high energy photons.

4.1 Plot of the deuteron polarization versus magnetic field for different doses of butanol. The polarization increases with magnetic field. The maximum polarizations were achieved for dopings of $2-3 \times 10^{15}$ electrons $/ \mathrm{cm}^{2} .58$

4.2 Polarization versus dose for a variety of deuterated materials. The maximum d-butanol polarizations were for materials doped with 2$3 \times 10^{15}$ electrons $/ \mathrm{cm}^{2}$. $\mathrm{CD}_{2}$ has a much broader maximum, but is centered around the same area. d-1-pentanol shares a similar optimal range. . . . . . . . . . . . . . . . . . .

4.3 The polarization as a function of time for the negative enhancement of Sample \#2. Note that the polarization builds quickly at first, but then rapidly levels off to $-66.3 \%$. . . . . . . . . . . . . . . .

4.4 Polarization versus incident dose. None of these materials approached the values obtainable in ammonia samples or their chemically doped counterparts. . . . . . . . . . . . . . . . 


\section{Chapter 1}

\section{Introduction}

Solid polarized nuclear targets have been used since the early 1960s to study particle interactions at laboratories around the world. The first low energy experiment with a polarized target was at Saclay [1]. That was followed by a high-energy experiment at Lawrence Radiation Laboratory at Berkley [2]. At first such experiments utilized hadron beams to study hadron-hadron interactions. Using incident particles such as $\pi$ and K-mesons scientists could extract the spin-sensitive part to prove or disprove different theories. With the advent of polarized nucleon beams nucleon-nucleon interactions could tell scientists about the specific details of the spin dependencies of the various interactions. Finally, the development of a polarized electron beam at the Stanford Linear Accelerator (SLAC) meant that deep inelastic scattering could be used to measure the spin content of the proton that is carried by the quarks.

Current experiments test the Standard Model of physics, which predicts that the valence quarks and the sea quarks are spin- $1 / 2$ objects that are bound by gluons, spin-1 objects. Spin phenomena are now being studied using polarized nuclear tar- 
gets at SLAC, Conseil Européen pour la Recherche Nucléaire (CERN), Bonn, the University of Mainz, MIT-Bates, and Thomas Jefferson National Accelerator Facility (JLab).

Studying the nucleons requires one to find suitable target materials. Since free neutrons do not exist for long in nature, the neutron present in the deuteron can act as one. Ideally, one would use $\mathrm{H}_{2}$ for protons and $\mathrm{D}_{2}$ for deuterons. Unfortunately, both are in their para-state with spin zero at low temperatures so they are not polarizable. Typical target materials, chosen for their radiation resistance, ease of handling and high ratio of free to bound nucleons, are diamagnetic materials like frozen alcohols and ammonia.

To polarize nuclear target materials they must be cooled to temperatures $\underset{\sim}{\prec} \mathrm{K}$ and placed in a strong $(2.5-7 \mathrm{~T})$ magnetic field. Cooling is typically done using a high power evaporation or dilution refrigerator. Even in such conditions the vector polarization of the proton will only be about $0.5 \%$. The Dynamic Nuclear Polarization (DNP) method is then used to enhance the polarization. This method, described in Chapter 2, requires that the material be properly doped with a paramagnetic centers.

Traditionally these centers have been introduced by two distinct methods. The first, chemical doping, is a highly successful technique. The second, irradiating the material, is also a viable technique and as been highly successful for doping materials such as ammonia.

Chemical doping is done using radicals such as EHBA, TEMPO and porphyrexide. It is only possible for substances that have a liquid phase slightly above room temperature. Once the dopant has been added to the material it is rapidly frozen to 
assure a homogeneous mixture of radical molecules in the host material. Chemically doped materials have the distinct advantage of being easily prepared and can have a short build-up time as well as high polarizations. Unfortunately, they generally have a weak resistance to radiation damage and are therefore only appropriate for low-intensity beam experiments.

Materials were first irradiated for use as a polarized target by Hwang and Sanders in 1960 [3]. Although they only achieved a polarization of $1.2 \%$ for the $\mathrm{CH}_{2}$, their irradiation was an important first step. Hill et al. furthered their efforts in 1966 using fast neutrons to irradiate $\mathrm{CH}_{2}$ [4]. With a magnetic field of $2.5 \mathrm{~T}$ and a temperature of $1.2 \mathrm{~K}$ they obtained a polarization of $10 \%$. These early efforts at irradiations were not expanded upon due to the success of chemically doped alcohols that were acheiving polarizations of up to $80 \%$.

The 1979 Coseners House Workshop reported that polarizations in excess of 90\% in irradiated $\mathrm{NH}_{3}$ and ${ }^{6} \mathrm{LiH}$ had been achieved. The 1980s saw huge improvements in the development of $\mathrm{NH}_{3}$ and $\mathrm{ND}_{3}$ targets. The biggest improvement came with the cryogenic system consisting of a $5 \mathrm{~T}$ magnet and $1 \mathrm{~K}$ refrigerator [5]. This decreased build-up times and increased polarizations when compared to earlier $2.5 \mathrm{~T} / 0.5 \mathrm{~K}$ systems.

Irritations are typically done at $90 \mathrm{~K}$ at a low energy electron beam. This first warm irradiation is then followed by a cold, in-situ irradiation done under liquid helium at $\sim 1 \mathrm{~K}$ during the nuclear physics experiment.

The physical processes involved when one irradiates a material vary considerably. A list of common materials and the paramagnetic radicals that are introduced into each one are given in Table 2.7 . 
As an example, when irradiating $\mathrm{LiH}$ the goal is to introduce F-centers that are vacancies in the anion sublattice that also include a trapped electron. This defect is effectively a hydrogen atom with an unpaired electron. The anion, since it is freed from its original lattice site, can be bound by another $\mathrm{H}$ atom to form an interstitial $\mathrm{H}_{2}$ molecule. There are several ways of doing this including exposing a material to UV-light, X-rays or additional coloration, but the most commonly used and most effective is to bombard the material with high energy particles. In the case of lithium hydride, the incoming particle ionizes an $\mathrm{H}$ atom. It becomes unstable and is ejected from its lattice site. This leaves a vacant site that can trap a free electron.

The probability of the ionized atom being ejected is lower at low temperatures. This means that the electron becomes trapped before a lattice defect can be produced. At higher temperatures the ion is more likely to be ejected from its lattice site leaving an anion vacancy that can produce an F-center. However, if the energy of the ionizing radiation is high enough the vacancy can be produced by a direct knock-out of the anion.

The optimal irradiation temperature is therefore a compromise between the ability of the ionized anions to leave their lattice sites to allow electrons to form F-centers yet not too warm as to allow the recombination of the F-centers with interstitial atoms and "healing" of the paramagnetic defects [6].

Target irradiations have been done using a variety of different configurations. Variables such as the length from the dewar wall to the target, the beam energy and the cryogenic material can have a major effect on the dose that is deposited on the material. A major goal of the work presented in this thesis is to normalize such target irradiations using EGS4, a Monte Carlo simulation tool used to determine the dose 
deposited in a given region.

A second goal is to predict the target heating due to electron and photon beams incident upon the target. Knowing the amount of heat deposited is an important consideration when determining the refrigeration system needed for a given experiment. The heat deposited by the beam must be less than than the cooling power of the refrigerator to ensure high polarizations. 


\section{Chapter 2}

\section{Solid Polarized Targets}

Solid polarized targets have long been used to study the internal structure of nucleons at high energy physics laboratories including JLab, Stanford Linear Accelerator (SLAC) and others. Target systems utilize low temperatures, high magnetic fields and microwaves to achieve polarizations of up to $100 \%$ for protons and $80 \%$ for deuterons using a process called Dynamic Nuclear Polarization (DNP). Low temperatures reduce the thermal motion of the system while the magnetic field splits the energy levels of the system by the Zeeman effect. Microwaves are then used to enhance the polarizations by DNP.

\subsection{Thermal Equilibrium Polarization}

The preferred method to achieve high nuclear polarization in target materials is to first produce high atomic polarizations that can then be transfered to nuclear spins. In practice, this is done by introducing paramagnetic radicals into the material prior to 
polarization. This is done because the nucleus has a much smaller magnetic moment than the electron making it easier to first polarize the electrons and then transfer that polarization to the nucleons via a dipole-dipole interaction.

When unpaired electrons are placed in a magnetic field $B$ in the $\hat{z}$ direction, they will tend to align themselves in the direction of that field. The degree of their alignment is quantified by a material's vector polarization, $P$, which is given by the following formula:

$$
P=\frac{\sum j_{i} N_{i}}{J \sum N_{i}}
$$

$J$ is the spin of the particle, $j_{i}$ is the projection of $\vec{J}$ onto the $z$-axis and $N_{i}$ is the number of nuclei in that state. The sum is over the $(2 J+1)$ orientations of the spin along the axis of the magnetic field.

This polarization can be calculated by statistical mechanics for different spin species. It is a function of the spin temperature, $T$, the magnetic moment of the species in question, $\vec{\mu}$, and Boltzman's Constant, $k$. To derive the equation for the polarization one begins with the partition function:

$$
Z=\sum_{j_{i}=-J}^{J} e^{\mu B_{z} j_{i} / k T}
$$

The polarization of the system is:

$$
P=\frac{\partial Z}{\partial(\mu B / k T)}
$$


simplifying the above equation leads to the Brillouin Function:

$$
P=\frac{2 J+1}{2 J} \operatorname{coth}\left(\frac{2 J+1}{2 J} \frac{\mu B}{k T}\right)-\frac{1}{2 J} \operatorname{coth}\left(\frac{1}{2 J} \frac{\mu B}{k T}\right)
$$

The two specials cases of spin- $\frac{1}{2}$ and spin- 1 are particularly important for the polarized target materials commonly used. In those instances:

$$
\begin{gathered}
P_{\frac{1}{2}}=\tanh \frac{\mu B}{k T}, \\
P_{1}=\frac{4 \tanh \frac{\mu B}{2 k T}}{3+\tanh ^{2} \frac{\mu B}{2 k T}},
\end{gathered}
$$

The spin temperature is not the more intuitive lattice temperature. It is a rigorously defined quantity that can be either positive or negative. Fortunately, over time, for a fixed magnetic field and lattice temperature, the spin temperature will be equal to the lattice temperature assuming there are no microwaves present. At that point, a measurement of the lattice temperature will also be a measurement of the spin temperature. At this, Thermal Equlibrium (TE) state, we can us Equations 2.5 or 2.6 to determine the vector polarization of the material.

Unfortunately, at typical laboratory conditions with magnetic fields of $5 \mathrm{~T}$ and temperatures around $1 \mathrm{~K}$, proton polarizations are only $\sim 0.5 \%$ which is inadequate for most applications. However, the electron polarization is $\sim 99.8 \%$. In order to achieve the high polarizations necessary for nuclear physics work one must use DNP, which is described below. 


\subsection{Dynamic Nuclear Polarization}

Dynamic Nuclear Polarization, (DNP), is a highly effective way to polarize materials to very high polarizations. In this technique, one uses the high gyromagnetic ratio, $\gamma_{S}$, of unpaired electrons, which are introduced by irradiation or chemical doping. Under typical laboratory conditions one can achieve nearly $100 \%$ electron polarization. With the aid of a strong microwave field at a frequency that is slightly different from the electron's Larmor frequency, $\omega_{s}=\gamma_{S} B_{0}$, one can transfer the electron's high polarization to the nuclear spins.

The simplest model for how this works is the solid state effect. First discovered in $1958[7,8]$, it is the basis of the DNP process. For clarity, the following discussion will be restricted to nuclear spin systems with $J=\frac{1}{2}$. A set of $N_{I}$ nuclear spins with Larmor frequency $\omega_{I}$ and $N_{S}$ electron spins with Larmor frequency $\omega_{S}$ will be considered to be in a magnetic field, $B$, and temperature $T$.

Such a system can be in one of four possible "pure" states. This is because Zeeman interactions between the magnetic moment, $\mu$, and the external magnetic field $B$ will split a system into $2 J+1$ sublevels. Those states, listed in increasing energy where the double arrow represents the spin of the nucleon and the single arrow represents the spin of the electron, are: $|\Uparrow \downarrow\rangle,|\Downarrow \downarrow\rangle,|\Uparrow \uparrow\rangle$, and $|\Downarrow \uparrow\rangle$. Between those four energy levels there are four allowed transitions. Two of these are nucleon spin flips ("NMR transitions") and two are electron spin flips ("EPR transitions"). The remaining two possibilities $|\Downarrow \downarrow\rangle \leftrightarrow|\Uparrow \uparrow\rangle$ and $|\Uparrow \downarrow\rangle \leftrightarrow|\Downarrow \uparrow\rangle$ are forbidden by dipole selection rules. However, the total Hamiltonian for the system has a spin-spin interaction term that allows mixing between states and allows previously forbidden transitions. 


$$
H=\overrightarrow{\mu_{e}} \cdot \vec{B}+\overrightarrow{\mu_{n}} \cdot \vec{B}+H_{s s}
$$

This term mixes the states in the following way where $c_{1} \approx 1$ and $c_{2} \ll 1$ :

$$
\begin{aligned}
& |\Uparrow \downarrow\rangle \rightarrow|A\rangle=c_{1}|\Uparrow \downarrow\rangle+c_{2}|\Downarrow \downarrow\rangle \\
& |\Downarrow \downarrow\rangle \rightarrow|B\rangle=c_{1}|\Downarrow \downarrow\rangle+c_{2}|\Uparrow \downarrow\rangle \\
& |\Uparrow \uparrow\rangle \rightarrow|C\rangle=c_{1}|\Uparrow \uparrow\rangle+c_{2}|\Downarrow \uparrow\rangle \\
& |\Downarrow \uparrow\rangle \rightarrow|D\rangle=c_{1}|\Downarrow \uparrow\rangle+c_{2}|\Downarrow \uparrow\rangle
\end{aligned}
$$

The probability of these forbidden transitions occurring is smaller than the allowed transitions by a factor of the dipolar mixing coefficient, $\epsilon$, squared. $\epsilon^{2}$ is about $10^{-4}$ for nucleons $1 \mathrm{~nm}$ away from the unpaired electron and it drops off as the sixth power of the distance [9]. This mixing, although several orders of magnitude less than EPR and NMR transitions, is what allows the transitions that were previously forbidden.

Assuming that a given material is properly prepared, when it is placed in a strong magnetic field and cooled to a low temperature, brute force polarization will force more of the population to be in state $|A\rangle$ than any other state. If one then irradiates the material with photons with the frequency $\nu=\left(\mu_{e}-\mu_{n}\right) B / h$ transitions from $|B\rangle$ 
to $|C\rangle$ will occur. Electron spins have strong interactions with the thermal motions of solids and decay rapidly through spin-lattice relaxation. This makes electron relaxation rates on the order of a few milliseconds. Since the rate for nucleons ranges from tens to hundreds of minutes, almost all of the $|C\rangle$ states will quickly decay into $|A\rangle$ states. By this method, anti-aligned nucleons will be converted into aligned nucleons. Likewise, irradiating the material with microwaves of frequency $\nu=\left(\mu_{e}+\mu_{n}\right) B / h$ will drive the $|A\rangle \rightarrow|D\rangle$ transition. Those nucleons will then decay to the $|B\rangle$ state. These processes are shown schematically in Figure 2.1.

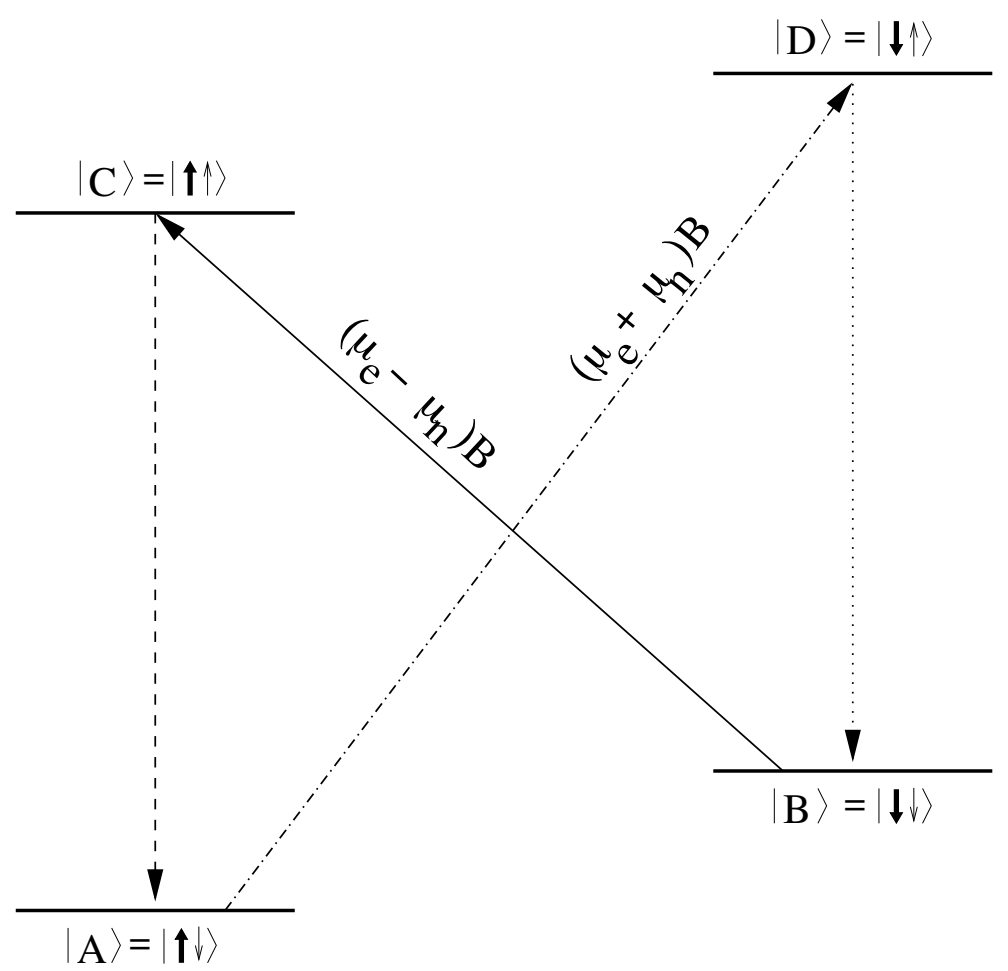

Figure 2.1: Energy level diagram for an electron-proton system in a magnetic field. Electron-proton systems driven from $|B\rangle$ to $|C\rangle$ with microwaves of frequency $\left(\mu_{e}-\mu_{n}\right) B / h$ will quickly decay back down to $|A\rangle$. Likewise systems irradiated with frequency $\left(\mu_{e}+\mu_{n}\right) B / h$ will be driven from $|A\rangle$ to $|D\rangle$ from where they will quickly decay to $|B\rangle$.

The previous section detailed the DNP process, but the coupling between electrons and nucleons only extends several angstroms. Spin diffusion is the process by which 
the polarization is spread throughout the material. Dipole-dipole interactions between nuclei transfer the spin of one nucleon to a neighbor in a flip-flop manner. This process spreads the polarization to regions of the material that cannot be directly polarized via the DNP process.

For most of the polarized target materials used today (such as frozen alcohols) the process of DNP is slightly different than the solid state effect described above. In these cases the concentration of electrons is high enough that the dipolar interactions of the electrons cannot be ignored so the Equal Spin Temperature theory is a more accurate description of the physics processes [10]. In this theory, the energy levels are not discrete, but rather a quasi-continuous band of states.

\subsection{Nuclear Magnetic Resonance}

The target polarization is measured via Nuclear Magnetic Resonance (NMR). This system works by inducing and detecting nuclear magnetic transitions. The rate of these transitions is proportional to the population differences between energy levels. One can therefore determine the polarization value.

When a rotating rf-field magnetic field is applied perpendicularly to an external field nuclear Zeeman transitions will be induced. The difference in rates of such transitions will indicate the polarization of the nucleons. In the UVA target the transverse magnetic field is generated by a current in a small coil that surrounds the material. This coil is part of the rf-resonant circuit that either absorbs or emits energy depending on the material's polarization. The static magnetic field is created by the target magnet. 
Under these conditions the magnetic susceptibility of the target material is:

$$
\chi(\omega)=\chi^{\prime}(\omega)+i \chi^{\prime \prime}(\omega)
$$

Where $\chi^{\prime}$ is the dispersive and $\chi^{\prime \prime}$ is the absorptive component. It can be shown that the integral over all frequencies of the absorptive component is directly proportional to the polarization [11]:

$$
P=\frac{2}{\mu_{0} \pi \hbar \gamma^{2} N I} \int_{0}^{\infty} \chi^{\prime \prime}(\omega) d \omega
$$

where $\gamma$ is the gyromagnetic ratio, $N$ is the spin density of the material and $I$ is the spin of the species being measured. For most species, the absorptive component is non-zero for a small frequency range around the Larmor frequency.

The target material acts as a dielectric inside the coil. Under these conditions the inductance becomes:

$$
L(\omega)=L_{0}\left[1+4 \pi \eta \chi^{\prime \prime}(\omega)\right]
$$

In this equation $L_{0}$ is the inductance of the coil without the dielectric, and $\eta$ is the filling factor. This, geometry dependent factor, describes the coupling of the inductor's field to the material. The impedance of the coil is therefore:

$$
Z(\omega)=R_{c}+i \omega L_{c}(1+4 \pi \eta \chi(\omega))
$$




$$
Z(\omega)=R_{c}+\omega L_{c} \cdot 4 \pi \eta \chi^{\prime \prime}(\omega)+i \omega L_{c}\left(1+4 \pi \eta \chi^{\prime}(\omega)\right)
$$

Therefore, measuring the complex impedance of the inductor, $Z(\omega)$, allows one to determine $\chi^{\prime \prime}(\omega)$. At UVA this step is done using the Liverpool Q-meter [12].

The Q-meter completes the LRC circuit by adding a capacitor and resistor in series with the inductance coil that surrounds the material. The design, which has the capacitor and resistor out of the cryostat, allows room temperature tuning. It is designed to measure the voltage change across the circuit as a function of frequency. The capacitance of the capacitor in the Q-meter is chosen such that the resonant frequency of the circuit is the Larmor frequency of the the spin species being studied.

The coil surrounding the target materials is connected to the Q-meter by means of a coaxial cable. This cable, often referred to as a $\lambda / 2$ cable, is invisible to the LCR circuit since it is chosen to be an integer number of half wavelengths at the Larmor frequency of the spin species being measured.

\subsection{Cryostat, Refrigerator and Magnet}

The achieve the low temperatures necessary for DNP, UVA uses a ${ }^{4} \mathrm{He}$ evaporation refrigerator capable of achieving temperatures of $\sim 1 \mathrm{~K}$. This refrigerator is housed in a specially designed Oxford cryostat that is similar to the SLAC cryostat shown in Figure 2.4. This cryostat has several cryogenic layers that are separated by vacuum ( $\sim 10^{-6}$ torr) regions to eliminate convective heating. The first layer is a $40 \mathrm{l}$ outside nitrogen reservoir that acts as a heat shield against the $300 \mathrm{~K}$ outer walls. The second layer is $104 \mathrm{l}$ liquid helium magnetic reservoir. Liquid is continuously drawn from this 


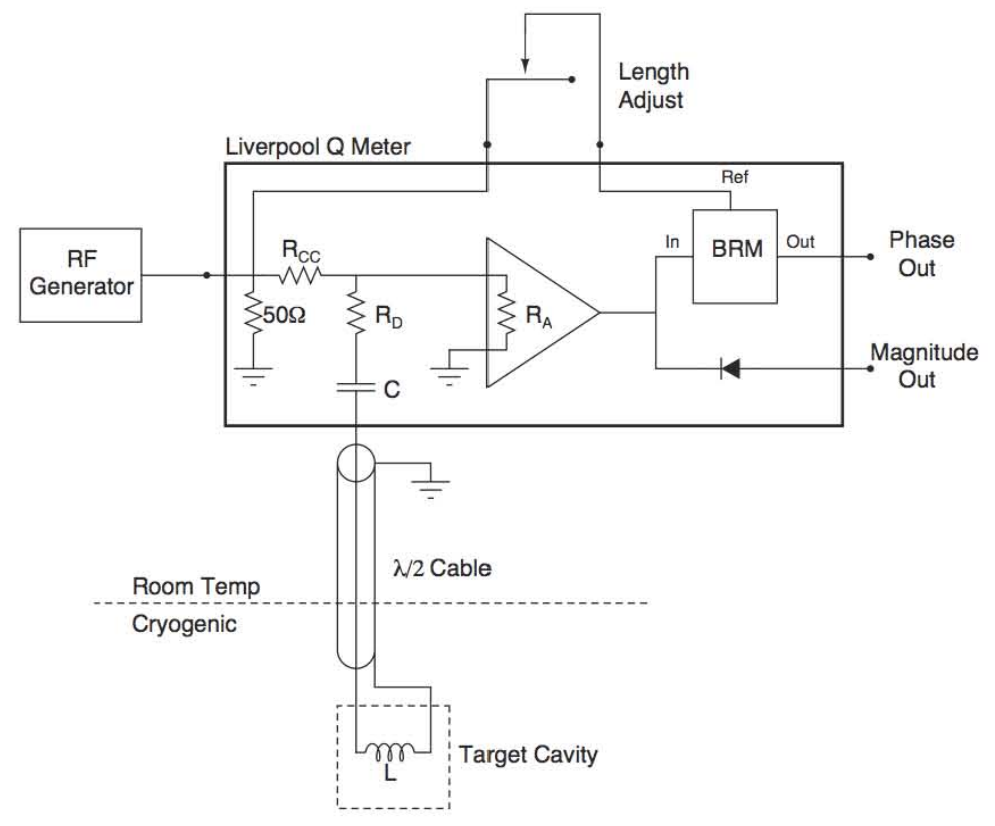

Figure 2.2: The Liverpool Q-Meter. Adapted from [14]

reservoir through a jumper line to feed the refrigerator.

The innermost layer of the cryostat contains the ${ }^{4} \mathrm{He}$ evaporation refrigerator, which is installed vertically. It includes two needle valves: a bypass valve that bypasses the main heat exchangers to initially cool down quickly; and a run valve that forces liquid through heat exchangers for optimal cooling. The system utilizes a series of both mechanical and Roots pumps to generate the power needed to achieve the low temperatures necessary for DNP and overcome external heat loads. The first stage of the pumping system is two mechanical pumps arranged in parallel. These first two pumps are linked in series to a small Roots pump that is in turn linked in series to two large Roots pumps connected in parallel. The mechanical pump alone is capable of reaching temperatures of $\sim 1.5 \mathrm{~K}$, while the extra pumping power of the Roots pumps are needed to achieve lower temperatures.

The super-conducting magnetic was built by Oxford Instruments and consists of a 
long solenoid. It is capable of reaching $8 \mathrm{~T}$, but it is usually run at $5 \mathrm{~T}$.

The $140 \mathrm{GHz}$ microwave tube used is built by Varian, Canada (now CPI) and is capable of providing up to $1 \mathrm{~W}$ of power to the material. Microwaves are sent to the material via a series of couplers and microwave guides. The power and frequency are measured continuously. The waveguide components outside the cryostat are rectangular in shape. Once inside the cryostat an oversized circular wave-guide is used to maximize the power that reaches the material. It is connected to a specially designed horn that broadcasts the microwave power directly onto the material.

The specially designed insert stick is shown in Figure 2.3. It is two meters long and contains two separate target cells each of which has its own NMR coil, temperature sensor and microwave horn. The $2.5 \mathrm{~cm}$ target cells can be filled with two different samples. Having two cells permits one to switch to the second cell once the material in the first has become radiation damaged. The stick itself is mounted to a mechanical piston allowing the user to move from one target to the next.

\subsection{Polarization Analysis}

Determination of the target polarization is done by integrating the area underneath the signal, which is calibrated by the Thermal Equilibrium (TE) signal. The first step is to measure the baseline of the NMR signal without the bump due to the presence of the polarized target material. To measure the baseline the magnet is moved so that it is slightly off-field, which makes it so the Larmor frequency of the target material is outside the sweep width of the NMR. The signal is then simply the parabola-like signal of the Q-meter. When the baseline measurement is completed, the magnetic 


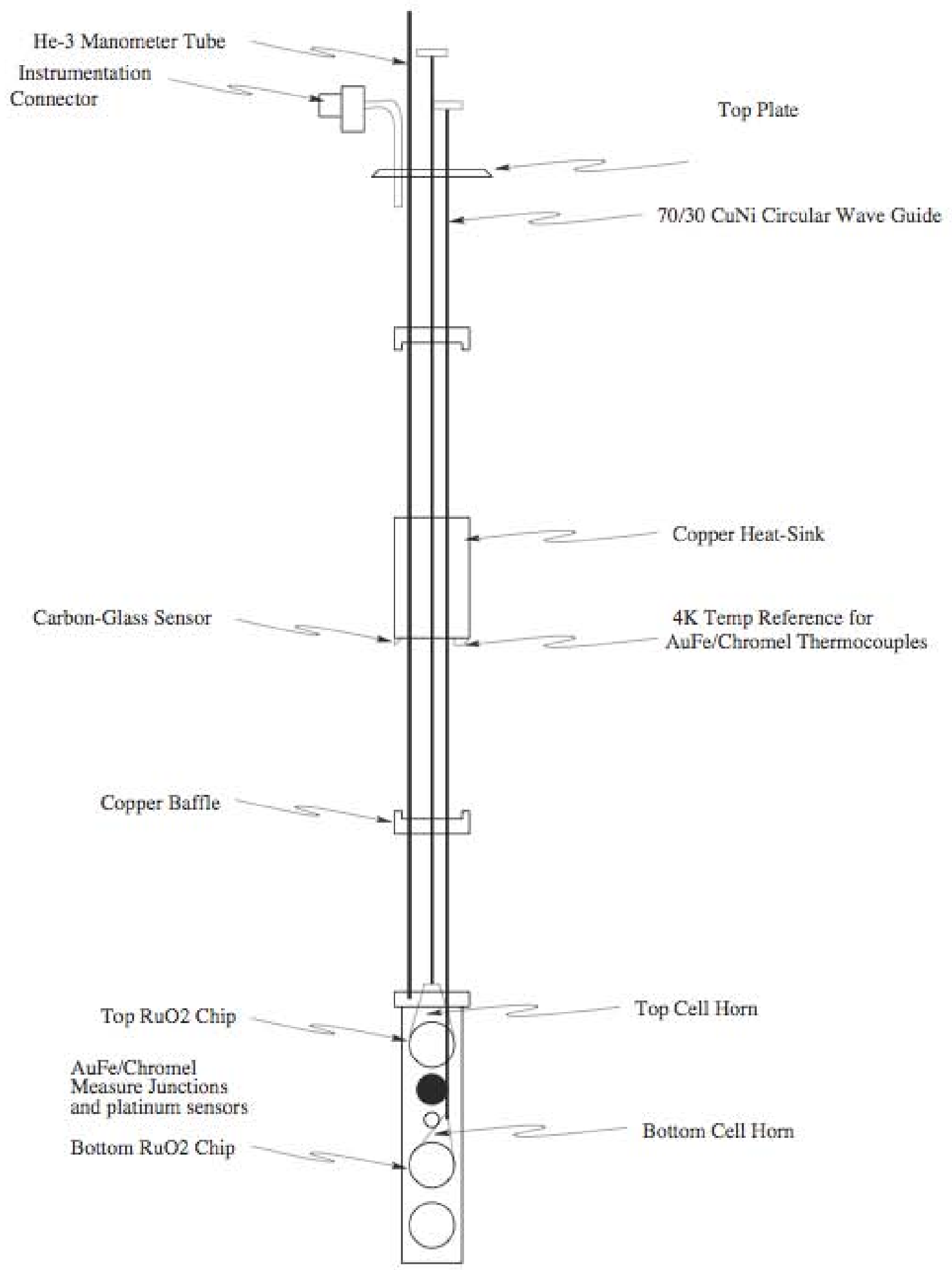

Figure 2.3: The insert stick. Not to scale. Adapted from: [13] 


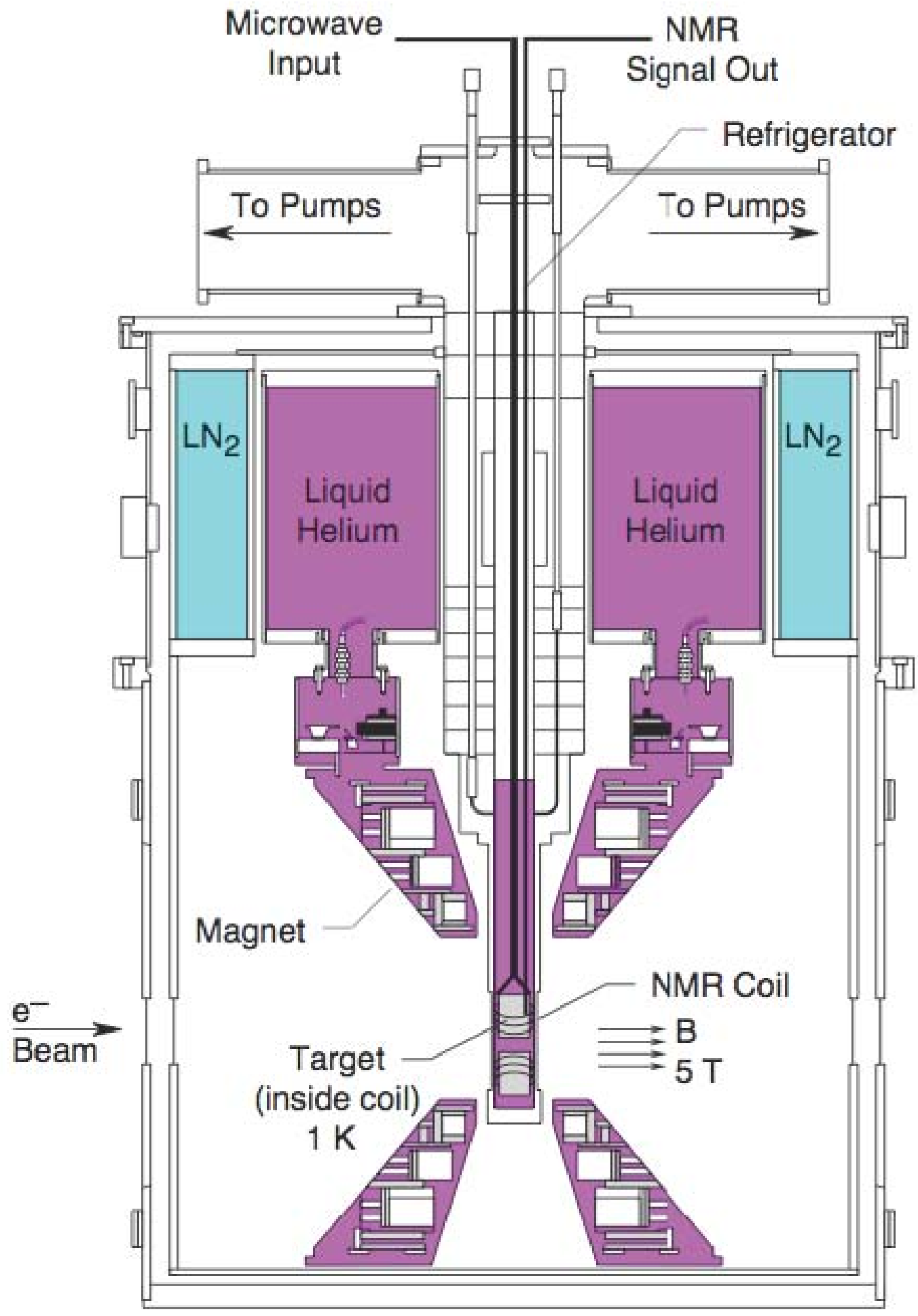

Figure 2.4: The SLAC Oxford Cryostat which can, in conjunction with the 4 He refrigerator cool target materials to $\sim 1 K$. The $L N_{2}$ shield on the outside acts as the outer heat shield. The magnet dewar keeps the magnet at $4 \mathrm{~K}$. Liquid is passed from this region to the refrigerator by means of a transfer tube (not shown). 
field can be restored.

Once the baseline measurement has been made, determining the polarization is done by the steps shown in Figure 2.5. The original signal acquired by the NMR DAQ system is shown in the upper left pane of that diagram. The baseline is first subtracted from the NMR signal to create the 'Subtracted Signal'. Temperature drifts in the Q-meters and the $\lambda / 2$ cable can cause drifts in the capacitance of the system that can change the shape of the Q-curve slightly. To account for this, the regions on either side of the peak are fitted to a polynomial. That polynomial is known as the 'Polysubtracted Signal'. That signal is then subtracted to give the final signal that is, in principle, solely a measurement of the material's polarization. The area, which is directly proportional to the material's polarization, is then calculated.

Calibrating the NMR system is done by first waiting for the lattice temperature to equal the spin temperature. The easiest way to determine when this occurs is to monitor the signal's area. The temperatures are aligned once it has reached its maximum area for a given constant lattice temperature. The time for this to occur varies drastically from material to material. Ammonia typically takes less than 30 minutes while LiD can take up to a day.

Once the two temperatures have become equal, the TE polarization can be easily calculated using Equations 2.5 or 2.6. Knowing the TE polarization allows one to easily calibrate the signal size to determine the calibration constant. The enhanced signal polarization can therefore be calculated in real-time using Equation 2.17.

$$
\text { Area } \times C C=\text { Polarization }
$$




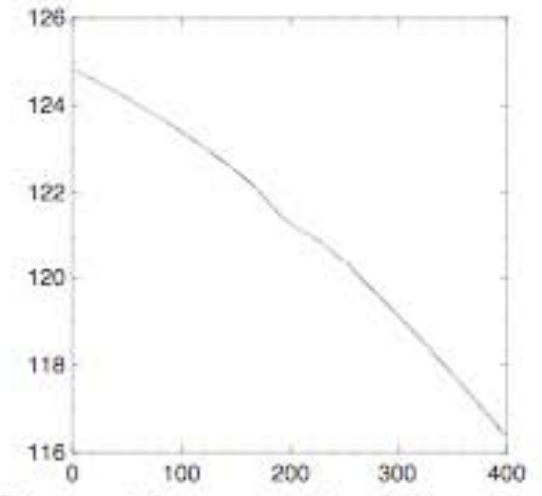

Signal is acquired by the NMR DAQ system

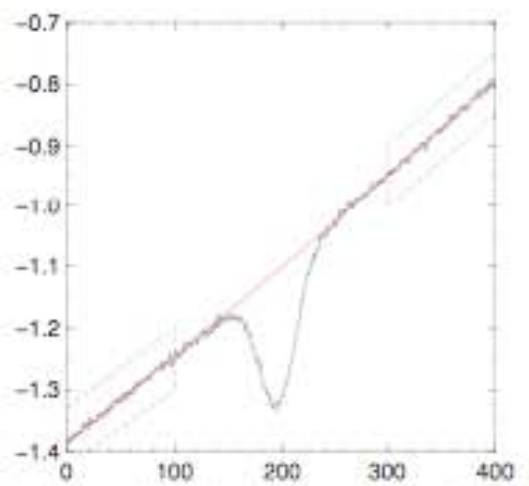

Polynomial is fit to wings of the signal

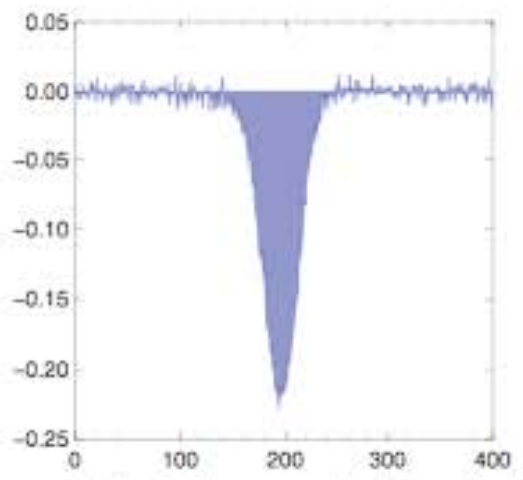

Signal is integrated, giving area

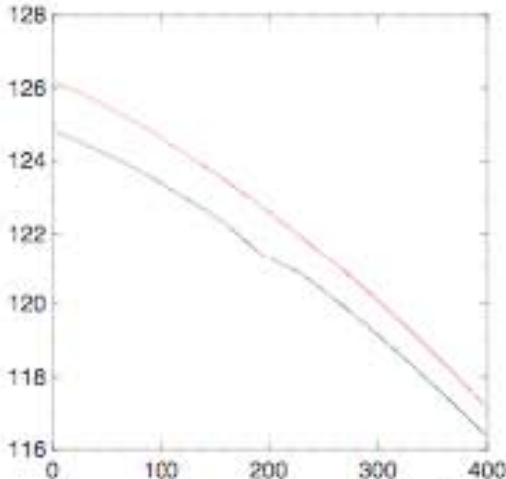

Baseline is subtracted from the signal

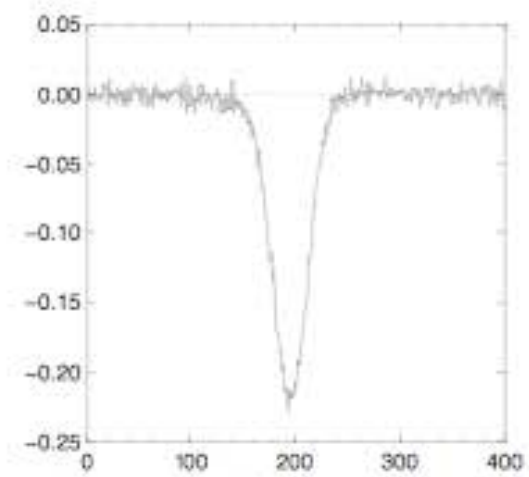

Polynomial is subtracted from the signal

\section{Area $\times \mathrm{CC}=$ Polarization}

\section{Calibrating the NMR system gives polarization}

Figure 2.5: The raw signal is shown in the upper left hand corner. First, the baseline is subtracted from that signal giving the 'Subtracted Signal'. A polynomial is fitted to the wings of it. That polynomial is then subtracted from the 'Subtracted Signal' giving the 'Polysubtracted Signal'. The resulting signal is then integrated. The polarization is directly proportional to the area underneath the 'Polysubtracted Signal'. The constant of proportionality is the called the calibration constant and the polarization can be calculated using Equation 2.17. Figure adapted from [14]. 
The spin 1/2 proton NMR signal approximates a Lorentzian with a FWHM of $\sim 60$ kHz. A typical signal is shown in Figure 2.5. To enable the baseline level of the signal to be established the frequency scan is normally made a factor of five higher than the width of the signal or about $300 \mathrm{kHz}$.

The deuteron signals look much different. A typical deuteron signal is shown in Figure 2.6. Since it is a spin 1 particle there are two spin transitions involved. This is because of the interaction of the quadropole moment of the deuteron with the electric field gradient of the molecule within which it resides. However, there are a few materials like LiD that only have a single peak because they are cubic symmetric with no electric field gradient. The resonance peak is $\sim 350 \mathrm{kHz}$ wide with a peak separation of $\sim 140 \mathrm{kHz}$.

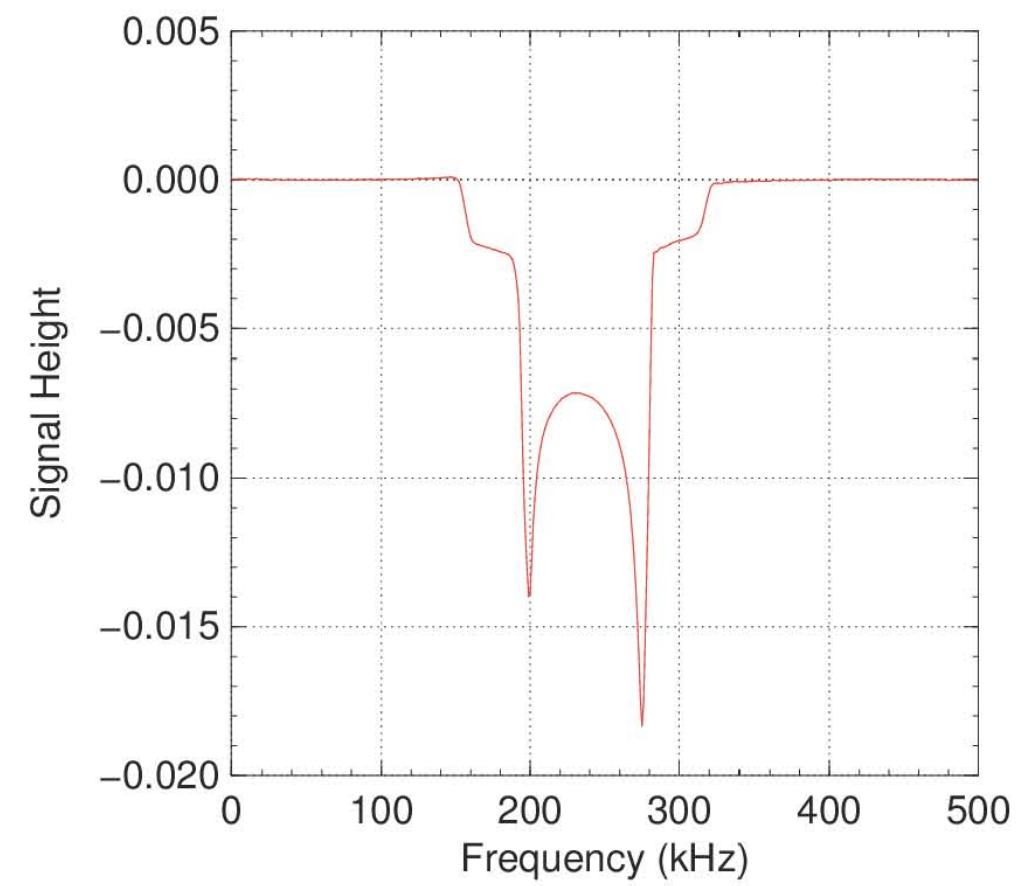

Figure 2.6: A typical deuteron signal. Note the characteristic double peaks associated with its quadropole moment.

Because of the lower line width and lower value of TE polarization the deuteron's 
signal size is much smaller. The small size of the deuteron TE means that it is often not practical to measures its area. In these cases the polarization can be measured using the ratio of peak heights in a typical deuteron signal. Essentially one is measuring the relative intensities of the $+1 \rightarrow 0$ and the $0 \rightarrow-1$ spin-state transitions. The vector polarization of a deuterated sample is then:

$$
P(D)=\frac{R^{2}-1}{R^{2}+R+1}
$$

where $\mathrm{R}$ is the ratio of peak heights. This method is only accurate for polarizations greater than $20 \%$.

\subsection{Polarization Error}

The polarization measurement of a given material is complicated by several factors that contribute to the overall error of the polarization measurement. The error comes mainly from the TE calibration. Effects like the movement of the target material around the NMR coil can be estimated by taking multiple TE calibrations. During a typical TE calibration 10 to $20 \mathrm{TE}$ measurements of the TE area are taken. These values are then averaged giving a mean and an error on that mean value.

Spin structure experiments are inclusive meaning that only the scattered lepton is detected. There is no way of knowing whether a given detected lepton was scattered from a polarized nucleon or from a residual proton in the deuteron target. Since the background nucleons can also be polarized they will contribute to the measured asymmetry. It is therefore necessary to know the composition and polarization of the background material. Fortunately, in the DNP process, the Equal Spin Temperature 
hypothesis can be used. This hypothesis assumes that, once thermalized, all constituents are at the same spin temperatures so the polarizations of each species only differs due to their individual magnetic moments. Most materials follow EST, but, for example, $\mathrm{ND}_{3}$ does not [15].

\subsection{Target Materials}

Target materials are chosen for maximum polarization and to the minimize radiation damage that degrades polarization. The most commonly used solid targets are diamagnetic materials including frozen alcohols, ammonia and lithium deuteride. These materials are then doped with paramagnetic radicals for the DNP process.

The success of the DNP process depends on finding a suitable combination of hydrogenor deuterium-rich material and a paramagnetic dopant. Ideally, the relaxation time should be on the order of milliseconds for the electrons and hundreds of minutes for the nucleons. An ideal proton material would be molecular hydrogen, but, unfortunately, it goes into a magnetically inert para-state that has spin zero at low temperatures. Fortunately, other materials have been found to be acceptable for nuclear physics experiments.

There are several important properties that any target material must have to be considered suitable for nuclear physics experiments. First, it must obtain a high degree of polarization. Second, its dilution factor, $f$, the ratio of free polarizable nucleons to the total number of nucleons, must also be high. Other considerations include practical constraints such as easy preparation and handling; having a short polarization build-up time; being highly resistant to radiation damage with the ability 
to repair such damage; and the presence of other polarizable nuclei.

Fortunately, there are several target materials that have all of the above properties and are regularly used in nuclear physics experiments. These materials include ${ }^{15} \mathrm{NH}_{3}$, ${ }^{15} \mathrm{ND}_{3}$, LiD, butanol $\left(\mathrm{C}_{4} \mathrm{H}_{10} \mathrm{O}\right)$. Other materials such as pentanol, ethane, methane, $\mathrm{LiH}$ and others have the potential and are an active area of research.

A disadvantage of using ammonia is the presence of polarized background nuclei ${ }^{14} \mathrm{~N}$ (spin-1) or ${ }^{15} \mathrm{~N}$ ( spin - 1/2). Since it is not possible to separately polarize the three hydrogens and the nitrogen the unwanted nitrogen contaminates the target material. It is therefore essential to know what its polarization is. The polarization of ${ }^{14} \mathrm{~N}$ is difficult to measure because it has a large quadrupole moment with peaks split $\sim 1 \mathrm{MHz}$ apart. Conversely, ${ }^{15} \mathrm{~N}$ has a single, narrow peak that is relatively easy to measure. Therefore, ${ }^{15} \mathrm{~N}$ is the preferred material for nuclear physics experiments.

\subsection{Target Material Preparation}

Target materials typically used are most easily bought in gas or liquid form. It is therefore necessary to freeze the materials before they can be irradiated. The method used depends on the initial state of the material.

Ammonia, ethane, methane and similar materials are bought in high pressure gas canisters. All materials can be prepared using the system described below, which was built originally as an ammonia freezing device.

Ammonia's freezing point is $\sim 195 \mathrm{~K}$ so it can easily be frozen at liquid nitrogen temperatures. The essential idea is that ammonia gas in the gas bottle will be cryopumped to the freezing chamber over the course of $\sim 15$ minutes. Once frozen, it can 


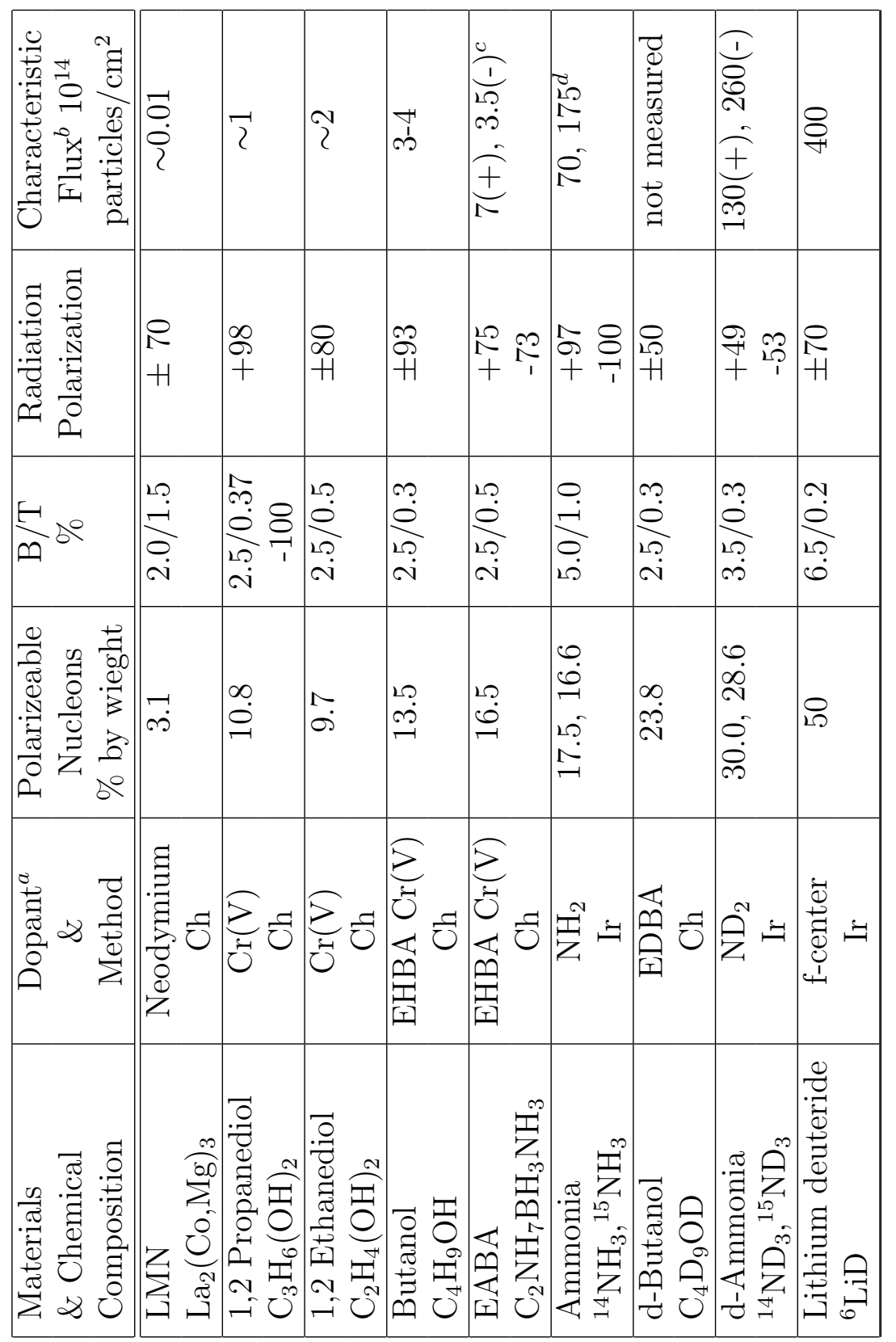

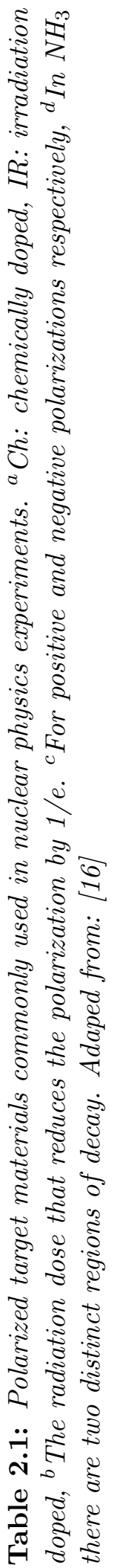


be extracted and crushed.

The newly designed system is a modification of an earlier system and is shown in Figure 2.7. It is made entirely out of stainless steel to resist corrosion. All joining sections are either SwageLock or pipe-thread assuring leak-tight performance.

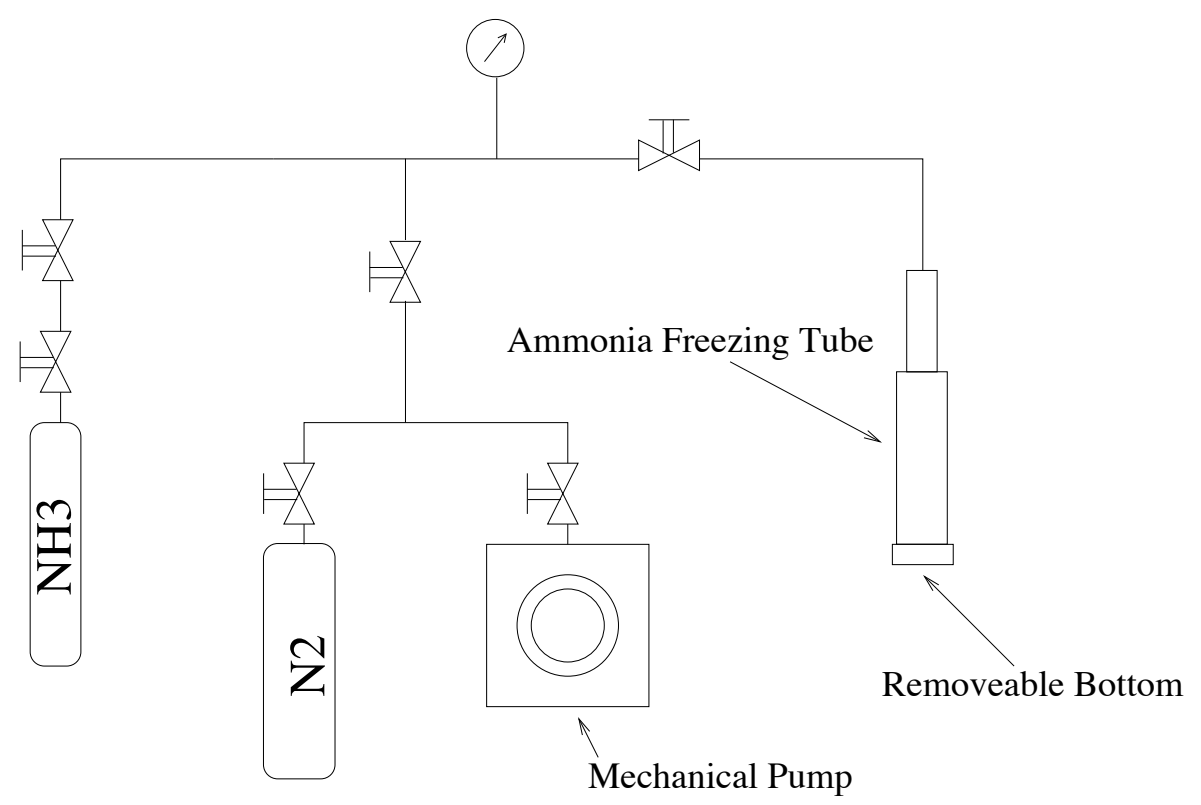

Figure 2.7: The newly-modified ammonia freezing apparatus. It is now constructed entirely out of stainless steel to resist corrosion. After the system is flushed with $\mathrm{N}_{2}$ and pumped down with the mechanical pump, ammonia gas is cryopumped from the gas bottle on the left to the ammonia freezing tube, which is immersed in a bath of liquid nitrogen.

The main freezing chamber, shown in Figure 2.8, is composed of a stainless steel tube with a Teflon-coated inside. It is $2.5 \mathrm{~cm}$ in diameter and about $23 \mathrm{~cm}$ long. It is composed of two separate parts for cryogenic reasons. The bottom is sealed with an indium seal to assure that it is leak-tight at low temperatures and to allow one to easily extract the ammonia slug.

The first step in the freezing process is to seal the freezing tube and check the system for leaks. Next one must pump and purge the system with nitrogen about three times. This is done to ensure that no impurities are frozen with the ammonia. Then 


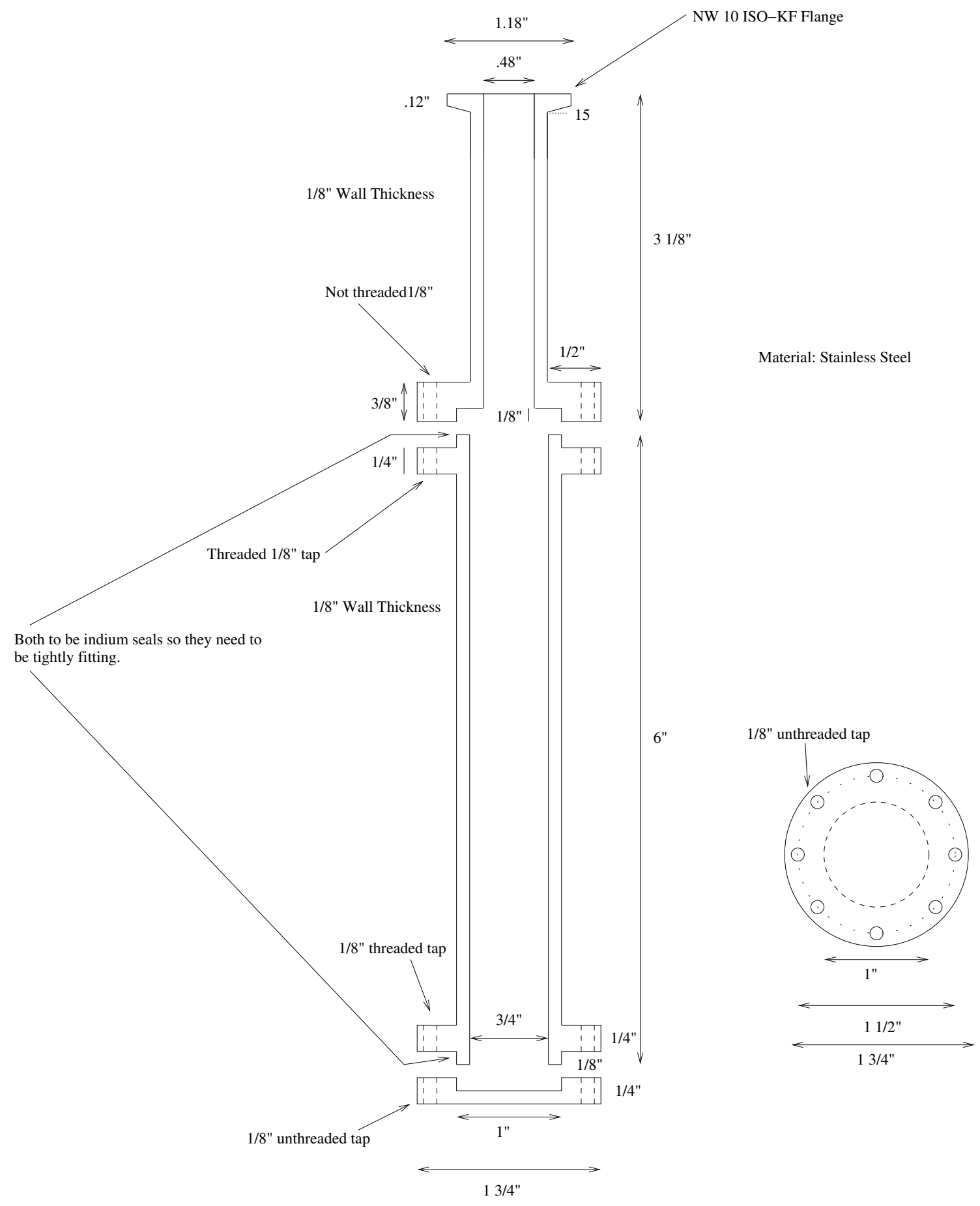

Figure 2.8: Newly designed ammonia freezing tube. The tube is made with stainless steel to be corrosion resistant. The end-cap is secured by eight screws and a indium seal to assure that it is leak-tight at low temperatures yet is easily unscrewed to allow the froozen slug to be removed. 
the freezing tube is cooled using a bath of liquid nitrogen to $\sim 77 \mathrm{~K}$. Once cooled, the ammonia gas bottle is opened up and the ammonia gas is cryopumped into the freezing tube. It typically takes about 15 minutes to cryopump enough ammonia to fill one $15 \mathrm{cc}$ bottle of frozen material. Once the desired amount of ammonia has been pumped from the bottle, the valve at the top of the bottle is closed and the remaining gas in the system is allowed to cryopump into the freezing tube for the same amount of time.

Once the material has been allowed to freeze for some time the indium seal is broken and the freezing tube is removed from the system. The bottom is taken off and it is suspended above a $\mathrm{LN}_{2}$ bath. After several minutes the material around the edges melts slightly and the ammonia slug falls into the bath. It is then carefully broken into smaller pieces that are approximately $1 \mathrm{~mm}$ in diameter with a pestle and some fine meshes.

Materials like pentanol and butanol are purchased in liquid form and are frozen with a bath of liquid nitrogen. The procedure for such preparation is to fill a burette with the material that is suspended over a bath of liquid nitrogen. The material then slowly drips into the bath and freezes on contact. The beads formed in this way are highly regular amorphous glassy spheres.

\subsection{Target Irradiations}

As described above, the DNP process requires that paramagnetic centers be deposited in the material. The radicals can be introduced into the target materials via two different methods. The first method is chemical doping using TEMPO, a stable 
nitroxyl radical. This method has been very successful for many materials.

Irradiating the material with electrons has also proven to be an effective method that introduces the radicals necessary for the DNP technique. Typically, this is done either at electron accelerator facilities, at high temperatures $(80-90 \mathrm{~K})$ or at low-temperatures $(\sim 1 \mathrm{~K})$ during nuclear physics experiments.

In low temperature irradiations the radicals necessary for DNP are introduced during the experiment. To produce the high radical densities needed this is only suitable for high-intensity beams of ionizing particles.

Studies have shown that some materials (most notably ammonia) that have been warm irradiated prior to the experiment perform better [17, 18]. Warm irradiations are typically done with beams that have energies of $20-300 \mathrm{MeV}$ and beam currents of 1-20 $\mu \mathrm{A}$. Irradiated samples, if kept under liquid nitrogen, can retain their radicals for several months to several years depending on the material.

$\mathrm{ND}_{3}$ requires a more complicated preparation method compared to $\mathrm{NH}_{3}$. To achieve high deuteron polarization one needs a low-temperature irradiation in addition to the high-temperature radiation. To achieve optimal results the electron flux needed is about $4 \times 10^{14}-10^{16} e^{-} / \mathrm{cm}^{2}$.

To perform the irradiations the material is inserted into a liquid argon bath and irradiated to the desired amount, typically $10^{15}-10^{17}$ electrons $/ \mathrm{cm}^{2}$. Liquid argon is necessary because such high energy electrons will create highly reactive radicals in liquid nitrogen. Argon does create $\mathrm{Cl}^{39}$ through proton knockout, but is it not produced in sufficient quantities to pose an imminent risk to those performing the irradiation as long as the beam energy is not sufficiently above the $16 \mathrm{MeV}$ production threshold. 
The irradiation dewar, shown in Figure 2.9, is constructed entirely out of aluminum with walls that are as thin as possible, especially in the area through which the beam passes. The outer region is pumped down using a diffusion pump to low pressure, while the inner can is filled with liquid argon. The irradiation stick is inserted into the can from the top and bolted down. It can be rotated $180^{\circ}$ to ensure a roughly uniform dose throughout the material.

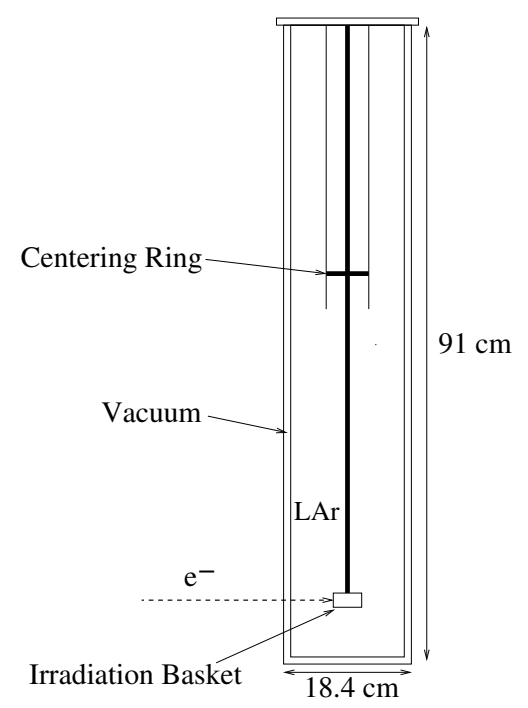

Figure 2.9: The material is held in the irradiation basket, which is immersed in liquid argon. The outer vacuum is held a high vacuum with a diffusion pump. Electrons, incident from the left have to travel through a total of $0.71 \mathrm{~cm}$ of aluminum and several centimeters of liquid argon before reaching the material. Figure is not to scale and dimensions are approximate.

The basket is attached to the end of the stick and is used to hold the target materials during an irradiation. It is held in place by two circular loops and is secured with a small screw and so it can be easily taken out to change materials. There have been several generations of baskets all with slightly different designs and sizes. The relative position and size of the three different generations of baskets are shown in Figure 3.1. The EGS studies, described in Chapter 2, prove that the exact geometries can have a large impact on the dose deposited. 
The UVA target group has irradiated materials at several facilities throughout the United States and Canada. Irradiations have been done at Bates Linear Accelerator Laboratory, the Monterey Naval Research Facility, the SUNSHINE facility at Stanford Linear Accelerator, the Saskatchewan Accelerator Laboratory and at Thomas Jefferson National Accelerator Facility. More recently, a series of irradiations have been done on many materials at the Medical and Industrial Radiation Facility (MIRF) at the National Institute for Science and Technology (NIST) in Gaithersburg, Maryland. Each facility operates at a different energy $(19 \mathrm{MeV}-300 \mathrm{MeV})$ and current $(1-20 \mu \mathrm{A})$. Furthermore, when a target material is irradiated in-situ it is done under liquid helium. These variables have a profound impact on the dose incident upon the target material. Such variables can be simulated using EGS4 which can allow one to normalize irradiations done under different conditions. EGS4 has the potential to compare directly the dose accumulated at other facilities and by other groups (e.g. the polarized target group at Bonn University, Germany).

Irradiations can take anywhere from 10 seconds to 3 hours depending on the desired dose, which differs for each material. The basket is always rotated at least once to permit even dosing.

Once irradiated, the material changes its color. The amount of color is related to the initial dose amount. Generally, the higher the dose the deeper the color is. However, it is not a linear relationship, since, although the color fades with time, the dose can remain for years if kept at liquid nitrogen temperatures. 


\section{Chapter 3}

\section{EGS4}

EGS4 is Monte Carlo simulation tool used to determine the dose deposited on a give material in a given volume [19]. At UVA it was used to simulate both target material irradiations and target heating during nuclear physics experiments.

\section{$3.1 \quad \mathrm{XYZDOS}$}

EGS4 allows the user to simulate the radiative transport of electrons, positrons or photons through elements, compounds or mixtures. The incident particles can have energies ranging from a few tens of $\mathrm{KeV}$ up to a few thousand $\mathrm{GeV}$. It does this by transporting particles through the material in random steps. EGS4 uses the following processes: Bremsstrahlung production, positron annihilation, Molière multiple scattering, Møller and Bhabha scattering, continuous energy loss applied to collisions to charged particle tracks between discrete interactions, pair production, compton scattering, Rayleigh scattering, and the photoelectric effect. 
EGS4 uses PEGS4, a stand-alone data preprocessing code. It creates piecewise-linear fits over different energies of the cross section and branching ratio data. It needs to be run only one time for each media. EGS4 itself is a package of subroutines that allow the user to determine the dose incident upon the material in a user-friendly environment. It uses the cross sections and branching ratios that were created by PEGS4.

The geometry can be specified by the user with the sub-routine HOWFAR and its subprograms. Magnetic and electric fields can even be added. The subroutine AUSGAB is used by the user to output the data.

For these studies XYZDOS was used to approximate the cylindrical basket volume filled with the material to be irradiated and the surrounding argon bath. XYZDOS is a convenient user code that allows the user to simulate the dose deposited for any geometry that is specified in cartesian coordinates. Its output is in arbitrary units of the dose deposited in a given voxel, which is a small cubic volume of material. The dose deposited can then be plotted as a function of any of the three coordinates to visually see where the dose is being deposited.

XYZDOS also gives the total amount of energy deposited in entire volume. This number can be used to calibrate the dose deposited in each region. Such simulations can be useful for both target heating questions as well as finding out the dose deposited at any given point in the material.

To simulate external magnetic fields EGS4 has an extension that can be used in conjunction with XYZDOS that allows the user to specify external magnetic field components in the $\hat{x}, \hat{y}$ or $\hat{z}$ directions. 


\subsection{The Radiation of Transport-Shower Processes}

As electrons or positrons traverse matter they lose energy by collision and radiation. At low electron energies the collision loss mechanism dominates, while at high energies radiation processes (bremsstrahlung) dominate.

When a collision occurs the atom is left either in an excited state or it is ionized. When ionized that ejected electron usually deposits a small amount of energy locally. Sometimes electrons are given enough kinetic energy to become a secondary particle called a delta-ray.

At high energies, a large fraction of the electron energy is spent on the production of high energy photons through one of the three main photo-processes: pair production, Compton scattering and the photoelectric effect. At high energies the production of electron-positron pairs dominates over Compton Scattering while the reverse is true at lower energies. These two processes produce secondary electrons that, through the bremsstrahlung process, generate an electromagnetic cascade shower. The third photon process, the photoelectric effect, along with multiple Coulomb scattering has a smaller effect on the shower. Both multiple Coulomb scattering and the Compton process cause a lateral spread. The above reactions cause an increased number of particles with decreasing average energy at each step of the process in the forward direction. At some point, the collision losses become more important so the energy of the primary electron is dissipated in the excitation and ionization of the atoms [19].

Solving the equations for the above processes analytically is prohibitively complicated. The differential equations, when solvable, are not that useful since they can

only be solved for a couple of special cases. The Monte Carlo technique allows the 
user to simulate all processes and accommodate any geometry. The random nature of Monte Carlo routines allows one to simulate the intrinsically random nature of electromagnetic showers.

\section{3 $\quad \mathrm{NH}_{3}$ Irradiation}

$\mathrm{NH}_{3}$ is frequently used in nuclear physics experiments, therefore its preparation is an important part of the work done at UVA. The following section aims better understand the irradiations done at the NIST MIRF facility. That facility has an electron linac that was run at an energy of $19 \mathrm{MeV}$ with a beam current of 4-20 $\mu \mathrm{A}$.

As was noted earlier, ammonia comes in gaseous form and is frozen using the technique described in Chapter 2. After freezing, it is irradiated with electrons at a particle accelerator. EGS4 was used to simulate the dose deposited and to normalize the current configuration with past generations of irradiation stick designs. As these studies will prove, even small variations in the design of the irradiation dewar and basket can have a large impact on the total amount of dose deposited.

As is described in Chapter $4, \mathrm{NH}_{3}$ irradiated in June 2005 was found to not polarize sufficiently. The maximum polarization was only $81.3 \%$. The problem was most likely that the material was under-irradiated. It was determined that the design of the baskets could have been the cause for this under-irradiation. The "New" basket design is compared to older designs in Figure 3.1. It is clear from Figure 3.1 that the "New" and "Middle" generation baskets are further back in the dewar and so do not receive nearly as much dose as the "Old" basket.

Previous NIST irradiations had used the "Middle" basket designs and so irradiation 
times had been determined using that configuration. But the "New" design holds the material approximately $0.5 \mathrm{~cm}$ further back than the "Middle" design. This means that incoming electrons will have to travel slightly further through the liquid argon. Since argon attenuates the electrons significantly it was theorized that not as much dose was being deposited into the ammonia. Even the $0.5 \mathrm{~cm}$ difference between the "New" and "Middle" generation baskets can make a significant difference.

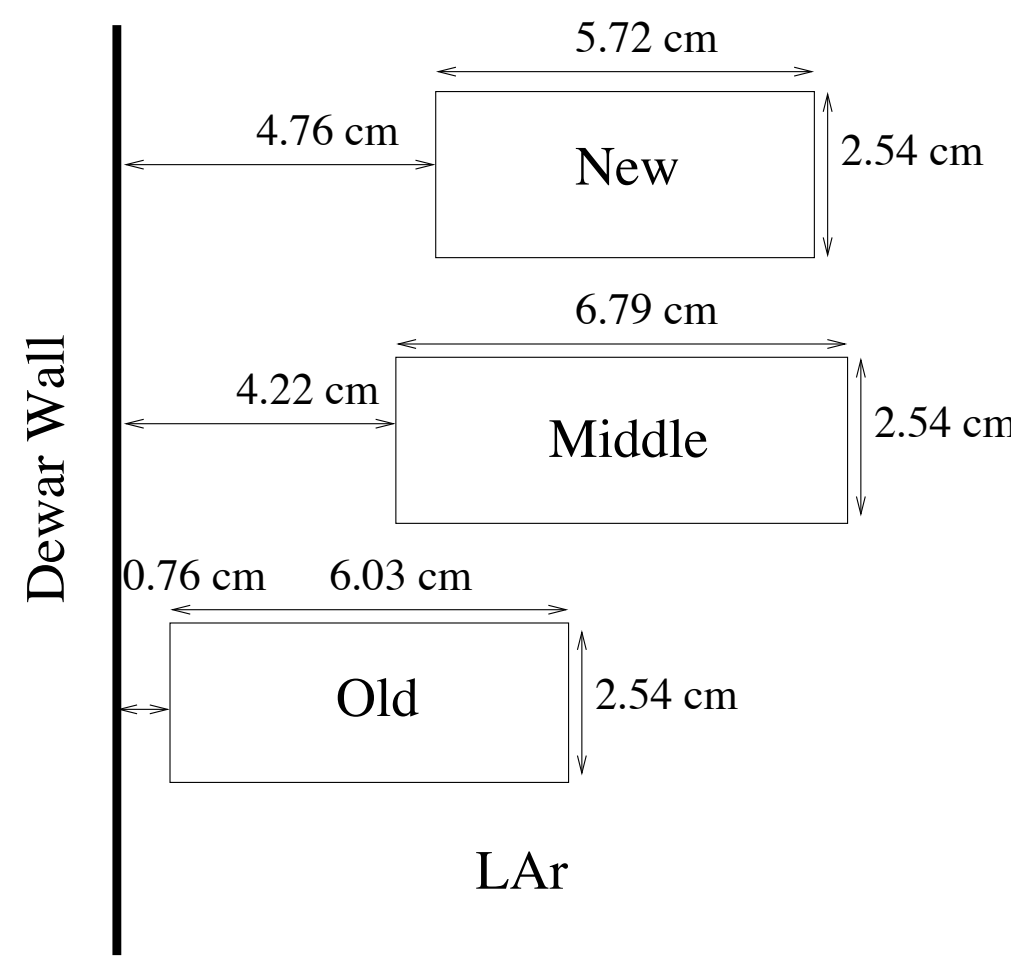

Figure 3.1: "New", "Middle" and "Old" basket designs and their positions relative to the dewar wall. The "New" and "Middle" baskets are similar in position, although the Middle one is slightly longer. The "Old" basket is pushed much further forward than the newer models. All are $2.54 \mathrm{~cm}$ in diameter.

To test this theory, an EGS4 simulation was done and the dose deposited as a function of $\mathrm{Z}$ was plotted and integrated to get the total energy deposited in a given region. XYZDOS does not allow the user to input more than two materials into a simulation. It was therefore necessary to compare three different test cases to determine what 
effect the different media have on the dose deposited. The five test cases were as follows:

1. $0.71 \mathrm{~cm} \mathrm{Al}+11.00 \mathrm{~cm} \mathrm{Ar}$

2. $0.71 \mathrm{~cm} \mathrm{Al}+11.00 \mathrm{~cm} \mathrm{NH}_{3}$

3. $11.71 \mathrm{~cm} \mathrm{NH}_{3}$

4. $4.56 \mathrm{~cm} \mathrm{Ar}+7.15 \mathrm{~cm} \mathrm{NH}_{3}$

5. $11.71 \mathrm{~cm} \mathrm{Ar}$

The $0.71 \mathrm{~cm} \mathrm{Al}$ represents the aluminum irradiation dewar wall and is the sum of the outer and inner vacuum walls. $11.71 \mathrm{~cm}$ is the total distance from the front of the irradiation dewar to the back of the furthest back basket design. $4.56 \mathrm{~cm}$ represents the average distance between the inside surface of the irradiation dewar and the front of the baskets.

These five different test cases are compared in Figure 3.2. From the diagram it is evident that the initial wall of aluminum increases the total energy deposited, but does not change the general shape of the curve. It is also clear that the case with just $\mathrm{NH}_{3}$ and the case with $\mathrm{Ar}+\mathrm{NH}_{3}$ are approximately the same. It can therefore be concluded that the aluminum wall contributes more to the differences in the simulations than the other materials. Therefore all further calculations will be done using the $\mathrm{Al}+\mathrm{Ar}$ configuration.

The most important result of these studies is that regardless of the configuration nearly all of the dose is deposited within the first $6 \mathrm{~cm}$ of the material. Figure 3.3, which plots $\mathrm{dE} / \mathrm{dz}$ vs $\mathrm{Z}$, depicts this clearly. This fact is essential since the target basket starts approximately $4.5 \mathrm{~cm}$ back. So, for the newer generations of basket 


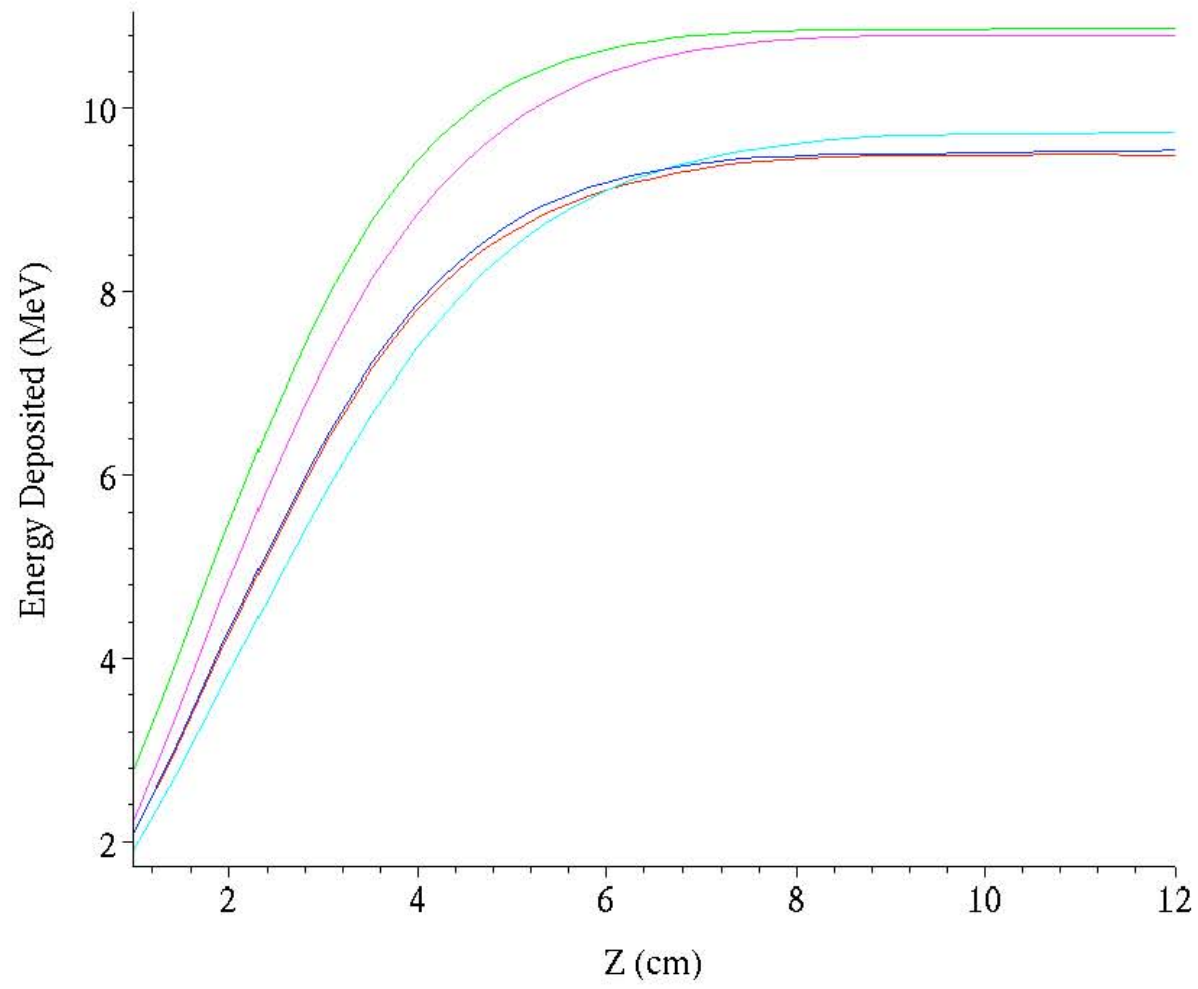

Figure 3.2: Total integrated energy deposited as a function of $Z$. Green $=A l+A r$, Pink $=\mathrm{Al}+\mathrm{NH}_{3}$, Light Blue $=\mathrm{Ar}$, Dark Blue $=\mathrm{Ar}+\mathrm{NH}_{3}$, Red $=\mathrm{NH}_{3}$. All curves have approximately the same shape, but those configurations with an initial aluminum wall have more energy deposited. The other materials make little difference in the amount of energy deposited. 
design, most of the dose is being deposited within the first centimeter or so. This is relevant to future designs of irradiation dewars and in comparing the dose deposited using other apparatuses or at other facilities.

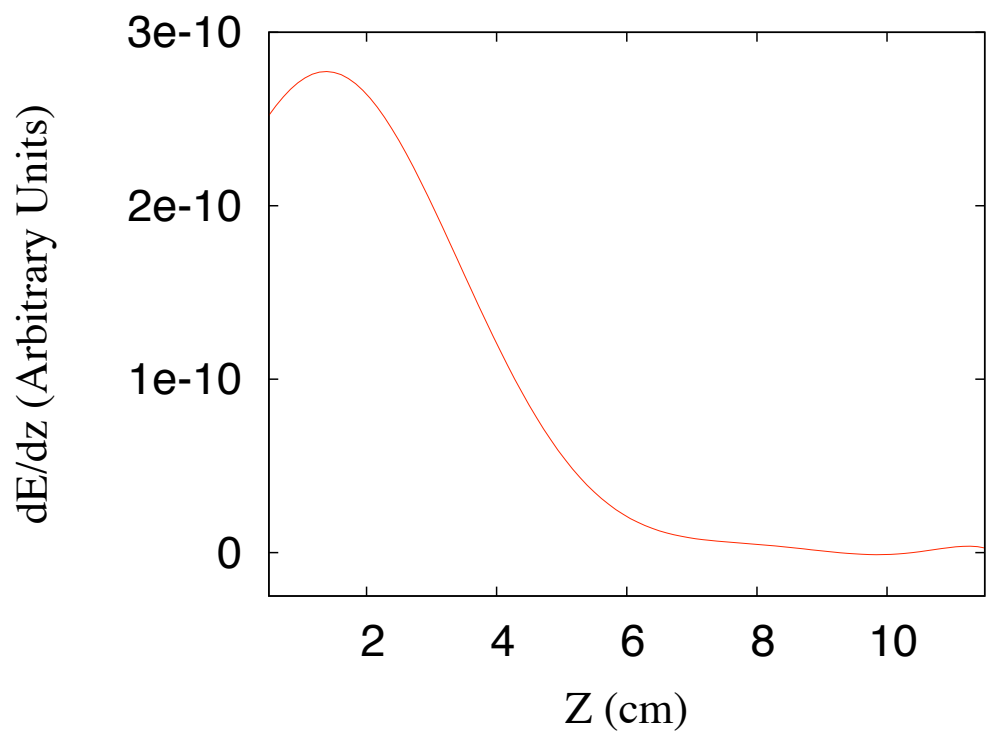

Figure 3.3: $d E / d z$ as a function of $Z$. Nearly all of of the dose is deposited within the first $6 \mathrm{~cm}$ of the target length.

Once the above test cases were concluded the dose deposited was integrated over the different basket lengths. As can be seen in Table 3.1 the energy deposited varies significantly between basket designs. In fact, the total energy deposited in the "Old" basket design is nearly 12 times the energy deposited in the "New" basket design. The differences between the "Middle" and "New" designs is not as big, but there is still a factor of 1.6 difference between the two. This Table indicates that the exact basket design, and its position in the dewar can be as important in determining the overall dose deposited as the beam current and the amount of time the beam is incident on the material. 
Table 3.1

\begin{tabular}{|l|c|}
\hline Basket & Deposited Energy $(\mathrm{MeV})$ \\
\hline \hline Old & 8.66 \\
Middle & 1.20 \\
New & 0.75 \\
\hline
\end{tabular}

Normalizing target irradiations run with different basket configurations can be done by taking the appropriate ratios of energies listed in Table 3.1. For instance, irradiations done using the "New" basket design need to be run for about 12 times as long as those done using the "Old" design.

\subsection{Duke University Laboratory}

Duke University's High Intensity Gamma-Ray Source (HIGS) produces polarized gamma rays by intra-cavity Compton backscattering FEL-photons from highly-relativistic electrons. This design optimizes the photon density. Experiments carried out using HIGS are interested in detecting low energy recoil protons in the target itself. This can reduce the background scattering and allow the measurement of many previously undetectable spin-dependent observables. To do this, a scintillating target can be used. The materials of choice are transparent vinyl-aromatic polymers with transparent luminescent additives.

The HIGS polarized target system is similar to the UVA system but with a dilution refrigerator to achieve temperatures of $\sim 50 \mathrm{mK}$ (in the frozen spin mode) and proton polarizations of $80-90 \%$ [20]. To determine the necessary cooling power an EGS4 simulation was run using a geometry that included a $0.254 \mathrm{~mm}$ aluminum wall with a $10 \mathrm{~cm}$ target length that was $1 \mathrm{~cm}$ in diameter. The scintillating target material 
Table 3.2

\begin{tabular}{|l|l|l|l|l|l|}
\hline $\begin{array}{l}\text { Energy } \\
(\mathrm{MeV})\end{array}$ & $\begin{array}{l}\text { EGS4 } \\
\text { Events }\end{array}$ & $\begin{array}{l}\text { Magnetic Field } \\
(\mathrm{T})\end{array}$ & $\begin{array}{l}\text { Field } \\
\text { Direction }\end{array}$ & $\begin{array}{l}\text { Deposited } \\
\text { Energy }(\mathrm{MeV})\end{array}$ & $\begin{array}{l}\text { Deposited } \\
\text { Power }(\mu \mathrm{W})\end{array}$ \\
\hline \hline 50 & $10^{6}$ & 0.5 & $\mathrm{X}$ & 1.449500 & 23.19 \\
50 & $10^{6}$ & 0.5 & $\mathrm{Y}$ & 1.447176 & 23.15 \\
50 & $10^{7}$ & 0.5 & $\mathrm{Z}$ & 1.666777 & 26.67 \\
50 & $10^{6}$ & 0.5 & $\mathrm{Z}$ & 1.674784 & 26.80 \\
50 & $10^{6}$ & 0.4 & $\mathrm{X}$ & 1.516178 & 24.26 \\
50 & $10^{6}$ & 0.4 & $\mathrm{Y}$ & 1.510553 & 24.17 \\
50 & $10^{7}$ & 0.4 & $\mathrm{Z}$ & 1.666300 & 26.66 \\
50 & $10^{6}$ & 0.4 & $\mathrm{Z}$ & 1.673800 & 26.78 \\
50 & $10^{6}$ & 0.0 & - & 1.6671505 & 26.67 \\
100 & $10^{6}$ & 0.5 & $\mathrm{X}$ & 1.981653 & 31.71 \\
100 & $10^{6}$ & 0.5 & $\mathrm{Y}$ & 1.979866 & 31.68 \\
100 & $10^{6}$ & 0.5 & $\mathrm{Z}$ & 2.201896 & 35.23 \\
100 & $10^{7}$ & 0.5 & $\mathrm{Z}$ & 2.203966 & 35.26 \\
100 & $10^{6}$ & 0.4 & $\mathrm{X}$ & 2.049953 & 32.80 \\
100 & $10^{6}$ & 0.4 & $\mathrm{Y}$ & 2.053653 & 32.86 \\
100 & $10^{6}$ & 0.4 & $\mathrm{Z}$ & 2.198080 & 35.17 \\
100 & $10^{7}$ & 0.4 & $\mathrm{Z}$ & 2.204479 & 35.27 \\
100 & $10^{6}$ & 0.0 & - & 2.21057 & 35.37 \\
\hline
\end{tabular}

was modeled by a hydrogen and carbon target.

Simulations were run for 50 and $100 \mathrm{MeV}$ incident photon beams with magnetic fields of $0.0,0.4$ and 0.5 T. For each field, simulations were run for the transverse $(\hat{x}$ and $\hat{y})$ directions as well as the longitudinal $(\hat{z})$ direction. Runs were done with $10^{6}$ as well as $10^{7}$ total incident photons to assure that an adequate number of trials was being performed. The results of these simulations are listed in Table 3.2. A photon flux of $10^{8}$ photons/second is used to simulate the HIGS beam.

The presence of a magnetic field decreases the amount of power deposited. It has a small effect in the $\hat{z}$ direction, while it has a larger effect in the $\hat{x}$ and $\hat{y}$ directions. With the magnet in the longitudinal direction the amount of power deposited is $\sim 0.5 \%$ less while it is $7-13 \%$ less in the transverse directions. The increase in energy 
deposited is most likely due to the geometry of the configuration. Since it is long (10 $\mathrm{cm})$ and skinny $(1 \mathrm{~cm})$ low energy electrons are more likely to be ejected out the sides of the volume.

As expected, there is little difference in the power deposited when the field is oriented in the $\hat{x}$ versus the $\hat{y}$ directions. However, when the field is oriented in the $\hat{z}$ direction $2-3 \mu \mathrm{W}(\sim 10 \%)$ more power is deposited when compared to the transverse directions.

It is clear that sufficient statistics were being run. The difference between photon fluxes of $10^{6}$ and $10^{7}$ incident photons is no more than $0.5 \%$.

When compared to the bigger field $(0.5 \mathrm{~T})$, the smaller magnetic field $(0.4 \mathrm{~T})$ allows more power to be deposited, particularly in the transverse directions. The difference in the transverse direction is $3.3-4.4 \%$, while the longitudinal differences are all less than $0.6 \%$.

The energy of the incoming photon beam has the biggest effect on the total power deposited. The $100 \mathrm{MeV}$ beam deposits 23.8 to $26.9 \%$ more power on the target volume than the $50 \mathrm{MeV}$ beam.

\subsection{Stanford Linear Accelerator}

The Real Photon Collaboration (RPC) was a group of scientists interested in creating polarized real photon beams with energies up to $45 \mathrm{GeV}$ using the LINAC at the Stanford Linear Accelerator Facility (SLAC). The idea was to use the LINAC as an electron source to create a collimated coherent bremsstrahlung polarized photon beam from a $1 \mathrm{~cm}$ diamond radiator. Using this technique, they hoped to achieve 
a quasi-monochromatic luminosity of about $5 \times 10^{10}$ photons $/ \mathrm{sec}$. A spectrum of the photon beam is shown in Figure 3.4. It is clear from these plots that there is a sharp peak around a central energy, but is non-zero at higher and lower energies. Three separate experiments were to use this beam including a measurement of the A dependence of $\mathrm{J} / \Psi$ photo-production; the gluon spin density within the nucleon; and the spin-dependent total cross section for circularly polarized photons absorbed on longitudinally polarized protons and neutrons $\left(\Delta \sigma^{\gamma N}(k)\right)[21,22,23]$.
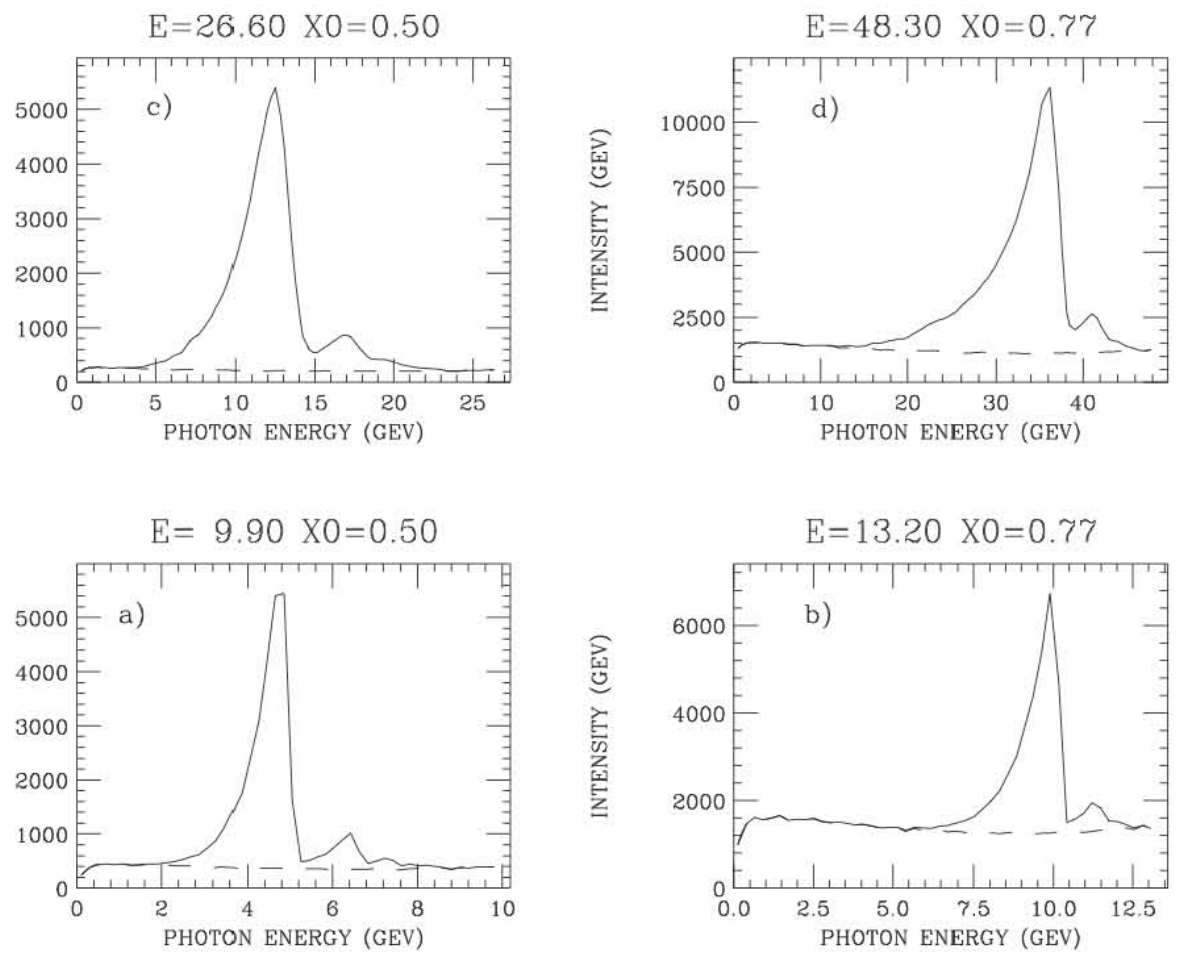

Figure 3.4: Intensity of a collimated coherent bremsstrahlung at four different energy levels. The resulting photon beam has a sharp peak around a single energy, but is non-zero are higher and lower energies. Adapted from: [22]

Two of these experiments: the gluon spin density, and the spin-dependent total cross section, were to use the polarized targets. To achieve the lowest possible temperature while maintaining adequate power a dilution refrigerator was to be used. This refrigerator was originally used at Conseil Européen pour la Recherche Nucléaire (CERN) 
in Geneva, Switzerland and is now being refurbished at UVA. However, the amount of cooling power necessary to overcome the power deposited by the incoming photon beam was not known accurately.

To quantify the target heating due to the incoming photon beam EGS4 simulations were run. These simulations were able to predict that the CERN dilution refrigerator, being refurbished at UVA, could provide the necessary cooling power.

As a photon travels through a material it is absorbed according to the following equation:

$$
\Delta I=I_{0}-I=I_{0}\left(1-e^{-z \mu}\right) \approx I_{0} z \mu
$$

where

$$
\mu=\mu(\rho, Z, E)
$$

$\rho$ is the density of the material, while $Z$ is the total number of protons and $E$ is the energy of the incident photon. At high energies pair production is the primary photon energy loss mechanism.

To approximate the cylindrical geometry, volume elements (voxels) of $0.159 \mathrm{~cm}^{3}$ were arranged in a $16 \times 16 \times 16$ cube. The circular $\mathrm{x}-\mathrm{y}$ plane was approximated by placing the sample into only those voxels that approximated a circular pattern.

The LiD sample was input by assuming that the lithium was composed of $96 \%{ }^{6} \mathrm{Li}$ and $4 \%{ }^{7} \mathrm{Li}$. Additionally a $60 \%$ packing fraction was assumed, making the additional volume $40 \%$ pure ${ }^{4} \mathrm{He}$. Using these numbers the density was calculated to be 0.545 $\mathrm{g} / \mathrm{cm}^{3}$ and the mixture was calculated to contain $9.16 \%{ }^{4} \mathrm{He}, 65.33 \%{ }^{6} \mathrm{Li}, 2.82 \%{ }^{7} \mathrm{Li}$ 
and $22.68 \%$ D by weight.

Other input parameters used include an electron energy interval of $1.511 \mathrm{MeV}$ to $45,000.511 \mathrm{MeV}$ and a photon energy interval of $1 \mathrm{MeV}$ to $45 \mathrm{GeV}$ (to simulate the $1 / \mathrm{k}$ photon spectrum). The global cutoff energies, below which particles were ignored, was set to be $10 \mathrm{KeV}$ for both the electrons and photons with a step size of $0.1 \mathrm{KeV}$. The beam was incident along the $\hat{z}$ direction with a spot size of $1.4 \mathrm{~cm}$ square.

To better understand the situation several different effects were considered. The first effect was that of the magnetic field. The high $(6.5 \mathrm{~T})$ magnetic field can help to contain those charged particles formed through pair production and other such mechanisms. Being contained, such particles can deposit more energy than usual into the volume under consideration.

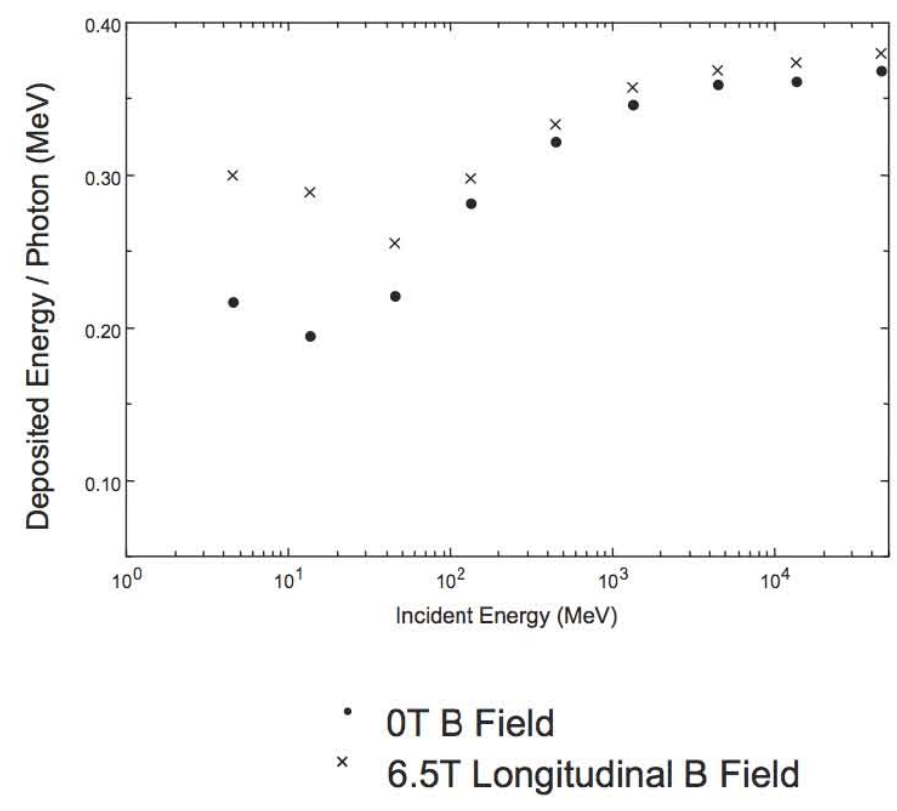

Figure 3.5: The deposited energy per photon as a function of the incident energy has a similar shape for both case of no magnetic field and a $6.5 \mathrm{~T}$ magnetic field. However, in the case of the 6.5 T magnetic field, the deposited energy is slightly less for low energies.

Figure 3.5 shows the energy deposited per photon as a function of incident energy. 
From that plot it is easy to see that the magnetic field has a large $(\sim 50 \%)$ impact at low energies, while that impact disappears for energies above $100 \mathrm{MeV}$. As expected the magnetic field helps to contain some of the particles created (especially at low energies) and therefore deposits more energy per photon.

The magnetic field direction is another important parameter that needed to be understood. Figure 3.6 shows the deposited energy as a function of incident energy. It is clear from this plot that, except at very high energies, much less energy is deposited in the case of the transverse magnetic field. Even at high energies, slightly more energy is deposited for longitudinal fields.

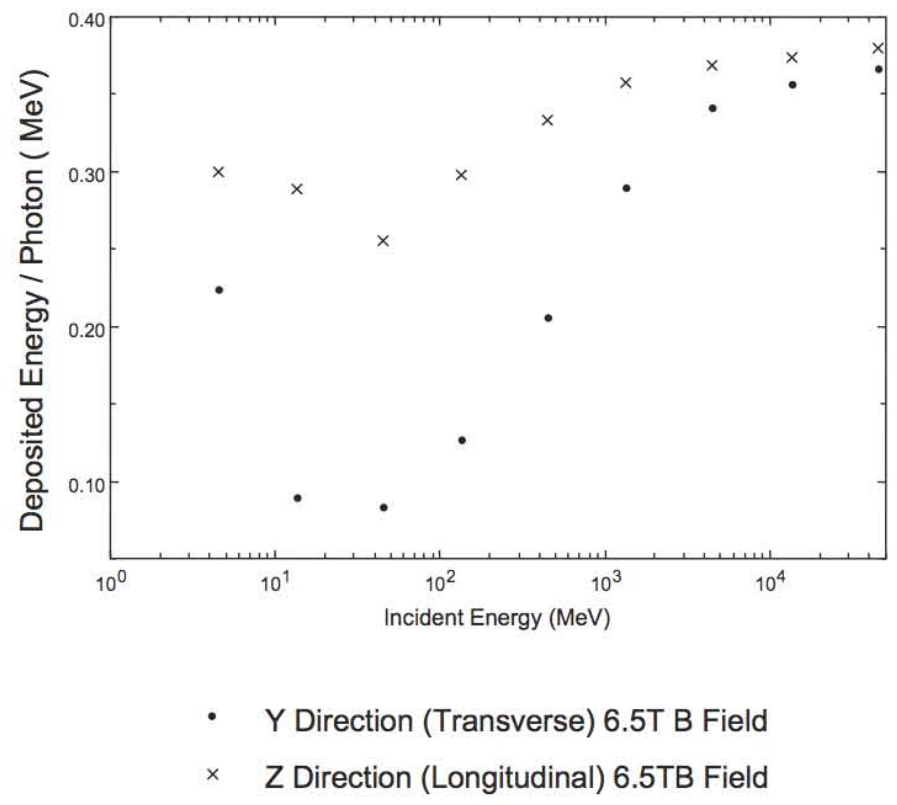

Figure 3.6: The energy deposited as function of incident energy is much less for the Transverse $(\hat{y})$ direction than it is for the Longitudinal $(\hat{z})$ direction for energies less than approximately $5 \mathrm{GeV}$

During the nuclear physics experiments the beam spot was to be a $0.5 \mathrm{~cm}$ circular area. To approximate this effect the simulation was run for the case of a square area and a no area "pencil" beam. As Figure 3.7 indicates, the spot size has little effect 
on the beam energy deposited.

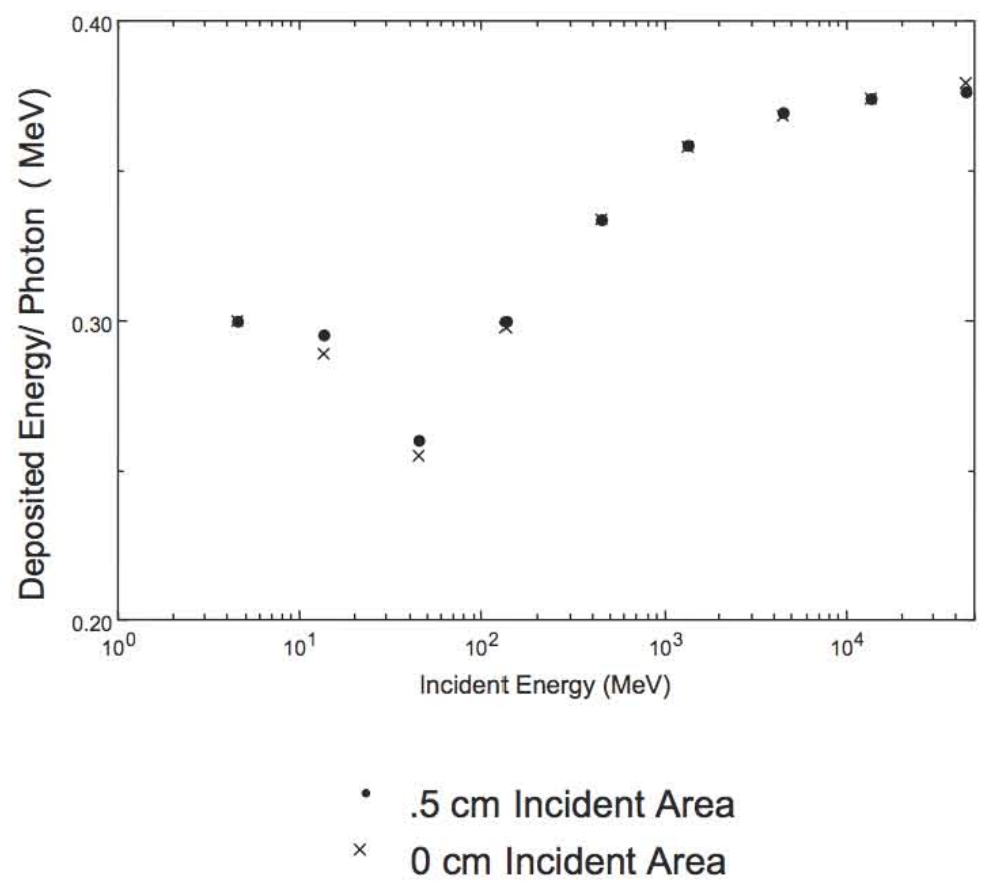

Figure 3.7: Two cases: one with a $0.5 \mathrm{~cm}$ area and one with a $0 \mathrm{~cm}$ incident area are shown to demonstrate the effects of spot size on the deposited energy. The size has little effect on the deposited energy per photon.

Precisely how the energy is deposited is an important piece of information. Figure 3.8 shows the energy deposited as a function of $\mathrm{Z}$, the longitudinal direction. Three different energies are shown: $4.5 \mathrm{MeV}, 450 \mathrm{MeV}$ and $45 \mathrm{GeV}$. For the two high energy cases (450 $\mathrm{MeV}$ and $45 \mathrm{GeV}$ ) the energy deposited is nearly linear as the photon shower moves through the material. On the other hand, the low energy incident photon levels off after about $3.5 \mathrm{~cm}$.

The energy deposited per voxel is graphed in Figure 3.9 where the $\hat{x}$ and $\hat{y}$ directions for a given $\mathrm{z}$ are graphed verses the deposited energy per photon. This plot is at $\mathrm{z}=$ $2.540 \mathrm{~cm}$ and is for a $45 \mathrm{GeV}$ beam. The plots at the other energies and regions are quite similar. It is clear from this plot that the energy is deposited almost entirely in 


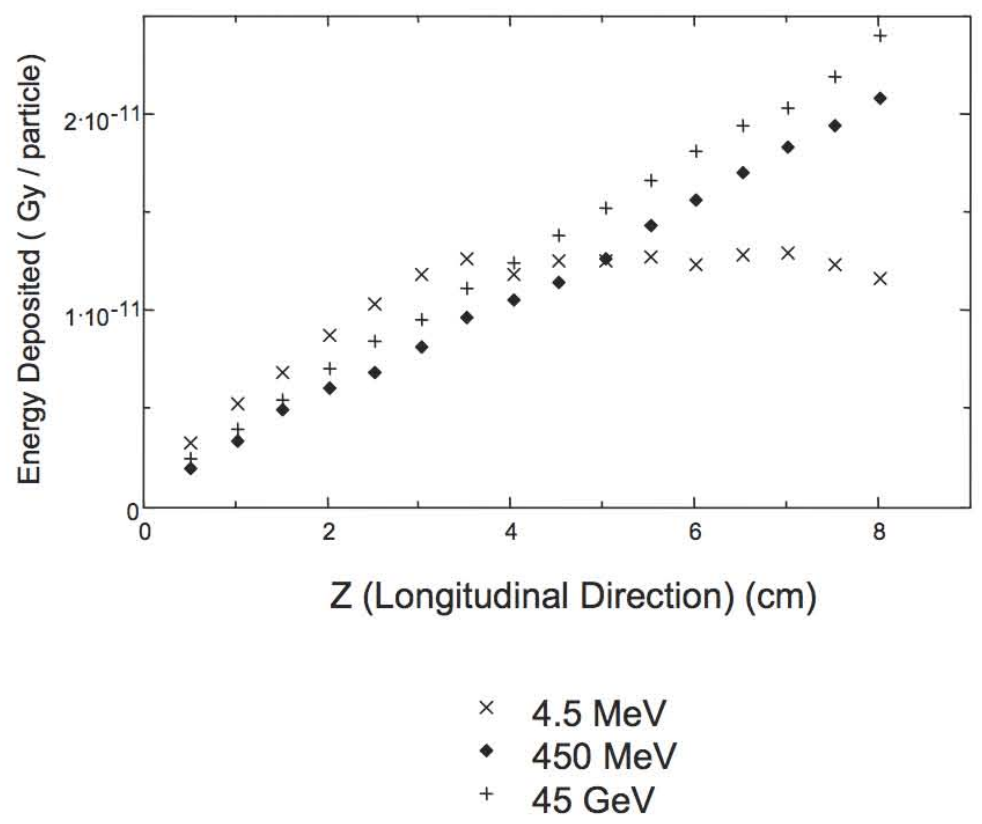

Figure 3.8: As a function of $Z$ the energy deposited is nearly linear for the $450 \mathrm{MeV}$ and $45 \mathrm{GeV}$ cases. It is only with the $4.5 \mathrm{MeV}$ case that the energy deposited decreases as the photons shower progresses through the material.

the regions where the beam was rastered since, on this scale, the dose is only visible in the regions from $0.477 \mathrm{~cm}$ to $2.063 \mathrm{~cm}$. Near the center of this region the dose is reasonably constant over the rastered region and rises to about $8 \times 10^{-12} \mathrm{gy} /$ incident particle. It was found that the dose became increasingly constant in the central region as the shower progressed deeper into the sample.

As discussed above, the spectrum of photons resulting from the bremsstrahlung has a sharp peak, but is non-zero over a much wider range. To determine the target heating that whole range needed to be used since peak energies only contribute a small fraction of the total number of incident photons. To do this, the spectrum was approximated by a $1 / \mathrm{k}$ spectrum. However, an important contributor to the spectrum is the LPM effect. First predicted by Landau and Pomeranchuk, the LPM effect suppresses bremsstrahlung radiation when its formation length becomes com- 


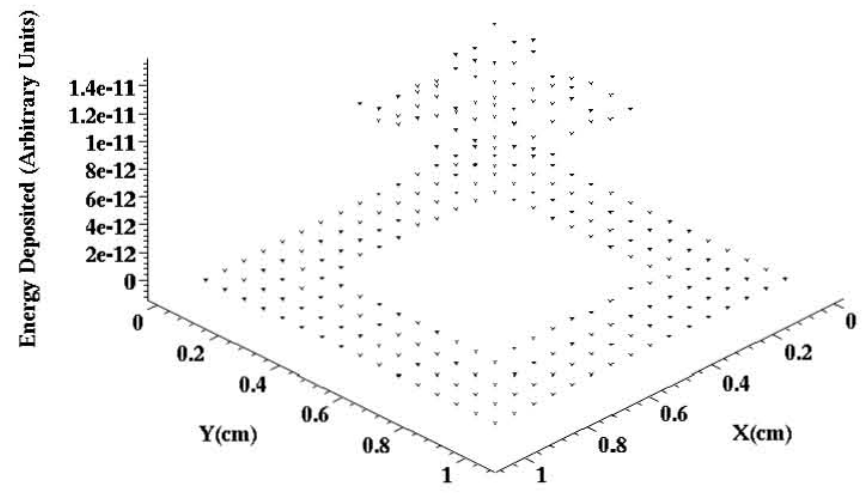

Figure 3.9: Dose deposited as a function of $x$ and $y$ in arbitrary units. From this plot it is easily seen the most of the dose is deposited in the rastered region.

parable to the distance over which the multiple scattering becomes important [24]. It reduces the photon flux below about $40 \mathrm{MeV}$ by about a factor of 25 making low energy photons less important. A normalized spectrum for an incoming beam of $10^{12}$ photons/second is shown in Figure 3.10. It is clear from this plot that the photon flux for low energy photons is significantly reduced below $40 \mathrm{MeV}$.

The actual power deposited (taking into account the LPM Effect) as a function of energy is plotted in Figure 3.11. Integrating the plot over all energies one finds that the energy deposited by low energy $(<40 \mathrm{MeV})$ photons is $1.2 \mathrm{~mW}$, while the high (>40 MeV) energy photons contribute $51.3 \mathrm{~mW}$. The $52.5 \mathrm{~mW}$ total is well within the capability of the dilution refrigerator. 
Photon Flux (10 12 Photons/Second) Including LPM Effect

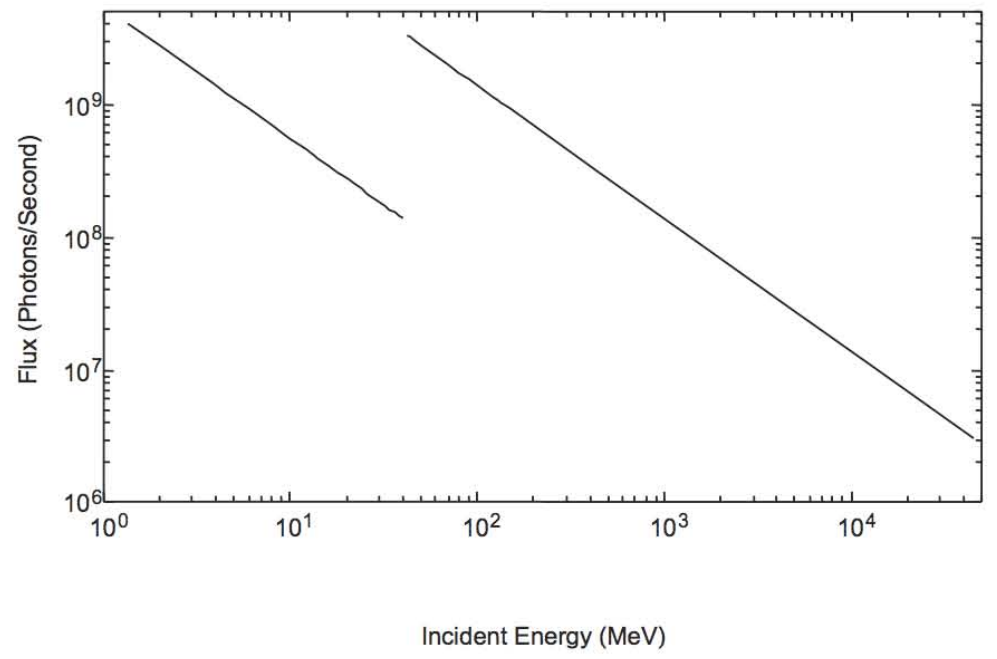

Figure 3.10: Photon flux with LPM effect. The $1 / k$ spectrum produced by the bremsstrahlung effect is reduced by a factor of 25 for photons below $40 \mathrm{MeV}$. The photon flux is normalized to $10^{12}$ photons/second.

Power Deposited (1011 Photons / Second)

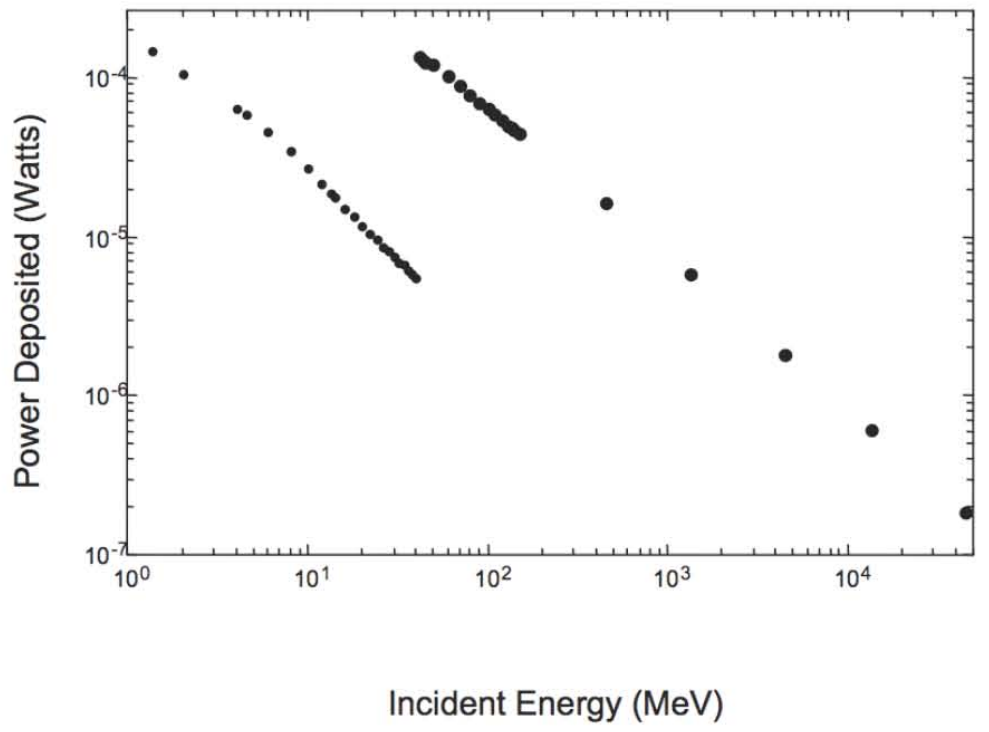

Figure 3.11: Total power deposited by the SLAC photon beam with the LPM effect included. Most of the deposited power is due to high energy photons. 


\section{Chapter 4}

\section{Polarization Measurements}

Careful measurements of irradiated proton and deuteron polarizations have been

made at UVA. Several deuterated materials were tested including D-buntanol $\left(\mathrm{C}_{4} \mathrm{D}_{9} \mathrm{OD}\right)$, $\mathrm{CD}_{2}$ and 1-d-pentanol $\left(\mathrm{CD}_{3}\left(\mathrm{CD}_{2}\right)_{4} \mathrm{OH}\right)$. The results of these studies indicate that deuterated materials perform better when they are irradiated while protonated materials achieve higher polarizations with chemical doping. The notable exception to this is $\mathrm{NH}_{3}$, which performs much better when irradiated.

For these measurements, the standard operating temperature of about $1 \mathrm{~K}$ was used with magnetic fields of $2.5 \mathrm{~T}, 5.0 \mathrm{~T}$ and $6.55 \mathrm{~T}$. A Carcinotron tube was used to provide the microwaves for the $2.5 \mathrm{~T}$ case. That tube operated at approximately 70 GHz. A $180 \mathrm{GHz}$ EIO tube was used with the $6.55 \mathrm{~T}$ magnetic field. 


\subsection{Deuterons}

D-butanol proved to polarize exceptionally well, particularily at high magnetic fields. It was irradiated with a dose of between 0.5 and $3 \times 10^{15}$ electrons $\mathrm{cm}^{-2}$. Figure 4.1 shows the polarization of D-butanol as a function of the magnetic field and dose. In most cases, polarization increased with magnetic field, with the one exception being the lowest dose. The positive enhancement was higher than the corresponding negative enhancement in all cases. The dose deposited has a smaller effect. The $3.0 \times 10^{15}$ dose proved to give the highest polarizations reaching a maximum of $62.5 \%$ and $-49.6 \%$ at $6.5 \mathrm{~T}$. The material with a dose of $2 \times 10^{15}$ electrons $\mathrm{cm}^{-2}$ had nearly identical results.

Figure 4.2 compares polarizations for d-butanol, $\mathrm{CD}_{2}$ and 1-d-pentanol at $5 \mathrm{~T}$ as a function of dose. $\mathrm{CD}_{2}$ had a broad maximum of $27.6 \%$ centered around $2 \times 10^{15}$ electrons $\mathrm{cm}^{-2}$. 1-d-pentanol proved to have polarizations nearly identical to dbuntanol.

Figure 4.1 also shows the data from some further measurements of d-butanol. In those measurements samples with doses of $2,2.5$ and $3 \times 10^{15}$ electrons $\mathrm{cm}^{-2}$ were repolarized for up to six hours. The $2.5 \times 10^{15}$ sample was polarized for two hours up to $+50.5 \%$ and $-47.6 \%$. Its polarization was measured using both the ratio and the TE method with nearly identical results. The 2 and $3 \times 10^{15}$ samples were also measured with build up times of six and three hours respectively. Approximately $97 \%$ of the final polarization was reached within three hours. 


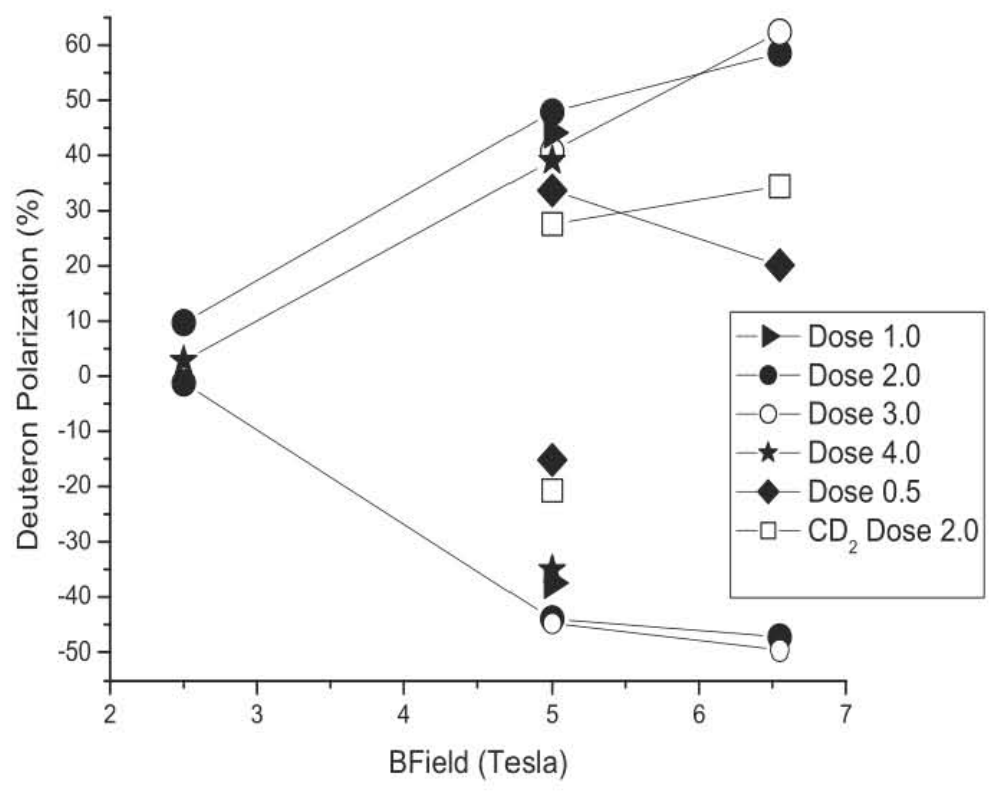

Figure 4.1: Plot of the deuteron polarization versus magnetic field for different doses of butanol. The polarization increases with magnetic field. The maximum polarizations were achieved for dopings of $2-3 \times 10^{15}$ electrons $/ \mathrm{cm}^{2}$.

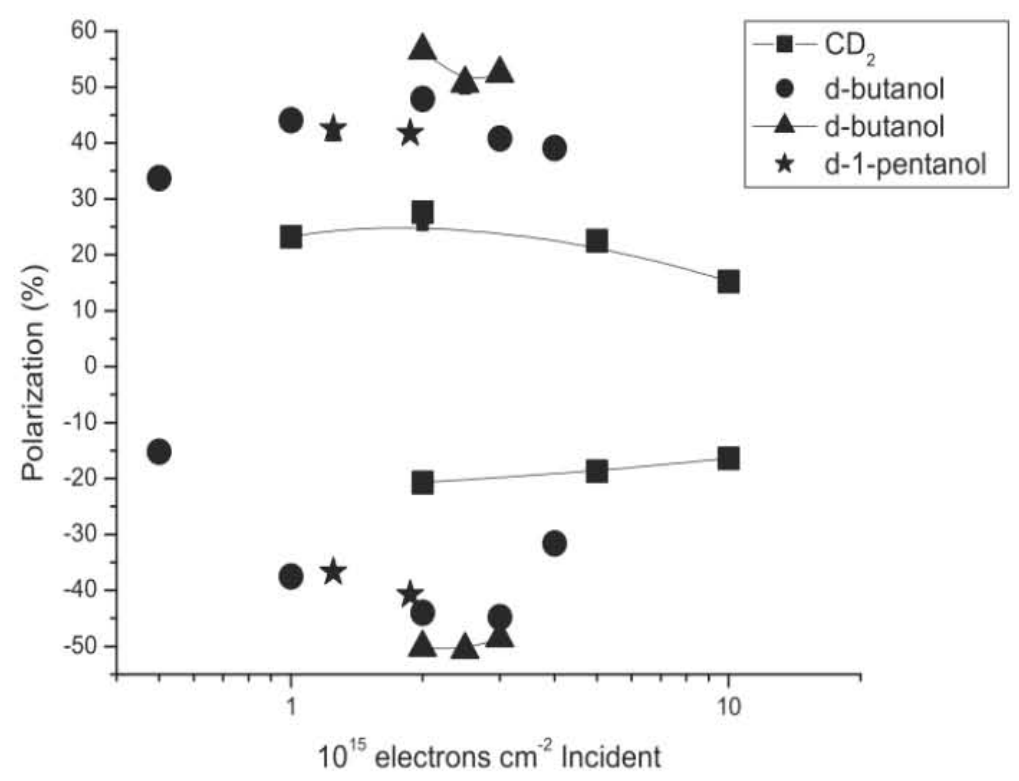

Figure 4.2: Polarization versus dose for a variety of deuterated materials. The maximum d-butanol polarizations were for materials doped with $2-3 \times 10^{15}$ electrons $/ \mathrm{cm}^{2} . C D_{2}$ has a much broader maximum, but is centered around the same area. $d$-1-pentanol shares a similar optimal range. 
Table 4.1: Polarizations for under-irradiated ammonia.

\begin{tabular}{|c|c|c|}
\hline Sample & Positive Polarization & Negative Polarization \\
\hline \hline 1 & $81.3 \%$ & $-73.2 \%$ \\
\hline 2 & $51.0 \%$ & $-66.3 \%$ \\
\hline 3 & $67.2 \%$ & $-77.2 \%$ \\
\hline 4 & $72.2 \%$ & - \\
\hline
\end{tabular}

Table 4.2: Polarizations for re-irradiated materials.

\begin{tabular}{|c|c|c|}
\hline Sample & Positive Polarization & Negative Polarization \\
\hline \hline 2 & $62.4 \%$ & $-71.0 \%$ \\
\hline Tempered \#2 & $70.7 \%$ & $-63.3 \%$ \\
\hline 5 & $92.9 \%$ & $-86.1 \%$ \\
\hline
\end{tabular}

\subsection{Ammonia}

As was previously discussed, the basket geometry can have a significant impact on the dose deposited into a given volume. Measurements taken in July-August 2005 revealed very poor ${ }^{15} \mathrm{NH}_{3}$ polarizations. These polarizations, listed in Table 4.1, were on the order of $50-80 \%$. They are far lower than the typical values of $>90 \%$. These samples were irradiated at NIST using the same setup as usual, with the exception of a newly designed basket. This basket, discussed in Chapter 2 is similar to, but is about $0.5 \mathrm{~cm}$ shorter than previous baskets. This difference, although minor, proved to have a large impact on the polarization of $\mathrm{NH}_{3}$.

The low polarizations seemed to indicated that the samples were inadequately irradiated. A typical polarization build-up is shown in Figure 4.3.

After the materials were newly irradiated using the "Old" basket design they were repolarized. The results of that series of polarization measurements are given in Table 4.2. Although polarizations were still not as high as expected, they were higher. The maximum polarization achieved by sample number 5 was $+92.9 \%$, which is a typical 
ammonia polarization. A second reason for the higher polarization was the discovery of some missing terminators from the NMR system. Once replaced the polarization jumped by about $10 \%$.

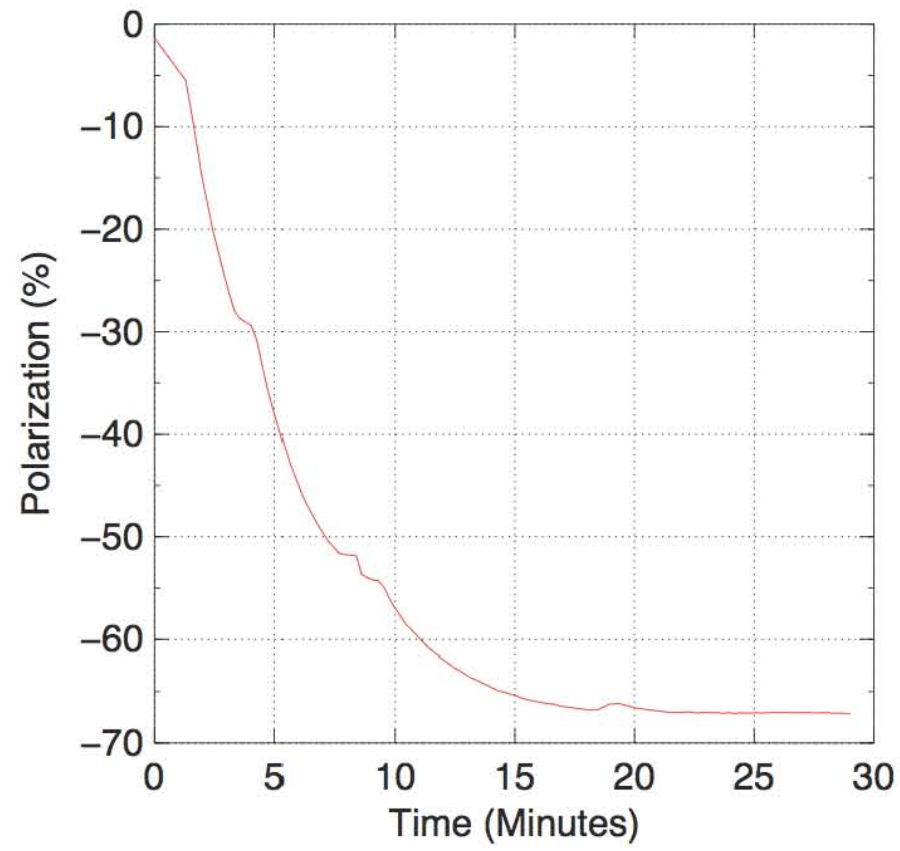

Figure 4.3: The polarization as a function of time for the negative enhancement of Sample \#2. Note that the polarization builds quickly at first, but then rapidly levels off to $-66.3 \%$.

\subsection{Other Protonated Materials}

Other protonated materials tested include $\mathrm{BH}_{3} \mathrm{NH}_{3}$, butanol, polyethylene $\left(\mathrm{CH}_{2}\right)$, borane ammonia $\left(\mathrm{BH}_{3} \mathrm{NH}_{3}\right)$ and 2-pentanol $\left(\mathrm{C}_{5} \mathrm{H}_{12} \mathrm{O}\right)$, the results of these polarizations are given in Figure 4.4. None of these materials polarized to a level approaching that of the chemically doped alcohols or irradiated ammonia. The results of polarization measurements as a function of dose are shown in Figure 4.4.

Borane ammonia and polyethylene have broad peaks of about $+25 \%$ and $-23 \%$ for 
doses centered around $2 \times 10^{15}$ electrons $/ \mathrm{cm}^{2}$. However, butanol and 2-pentanol have much narrower peaks centered around the same dose. Their polarizations reached a maximum of around $\pm 40 \%$.

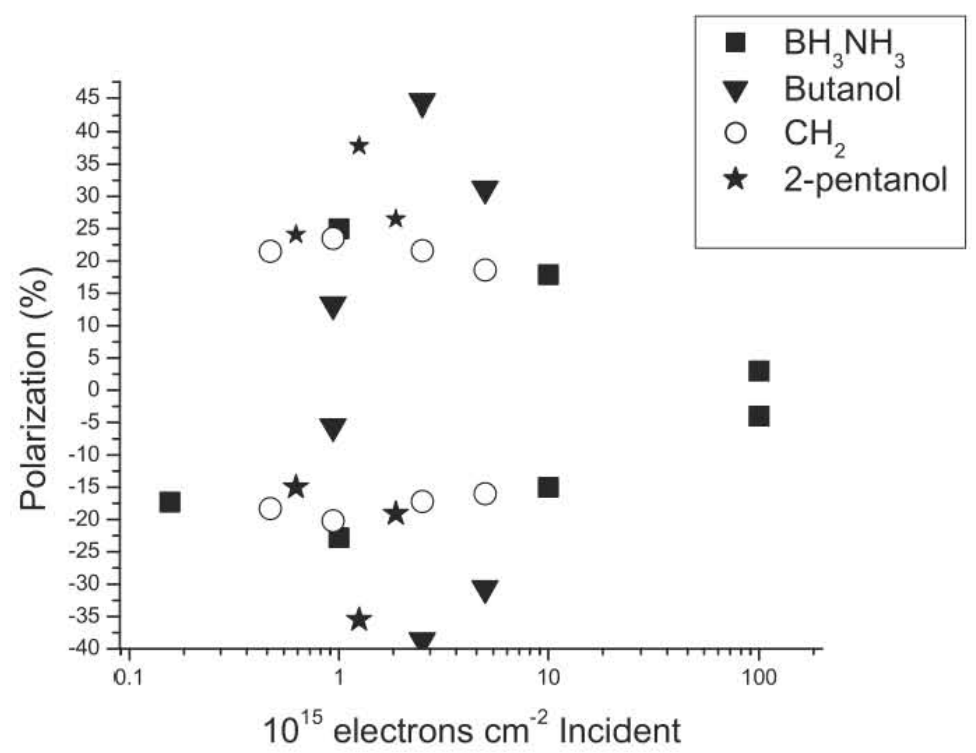

Figure 4.4: Polarization versus incident dose. None of these materials approached the values obtainable in ammonia samples or their chemically doped counterparts.

\subsection{Conclusions/Outlook}

This thesis used EGS4, a Monte Carlo simulation tool to both predict target heating during nuclear physics experiments and the dose deposited upon a sample during its irradiation.

The target heating simulations will be useful to scientists wishing to predict the amount of cooling power needed for a given refrigerator and target. Such simulations can easily be run for any particular configuration. 
The target irradiation simulations show that the exact geometry of the target material to be irradiated can have a significant impact on the amount of dose deposited into a given volume. Moving a target basket back by as little as $0.5 \mathrm{~cm}$ can make a large difference in the amount a target is irradiated. Scientists will now be aware of these issues and can run simulations specific to their irradiation dewar design. This will allow them to compare irradiations done at a particular facility to irritations done at other facilities or under differing conditions. 


\section{Bibliography}

[1] A. Abragham et al., Phys. Lett. 2:310 (1962).

[2] O. Chamberlain et al., Phys. Lett. 7:293 (1963).

[3] C. F. Hwang and T. M. Sanders, Helv. Phys. Acta Suppl. VI, 122 (1960).

[4] D. Hill et al., Phys. Letts., 23,63, (1966).

[5] D. G. Crabb, C. B. Higley, A. D. Krisch, R. S. Raymond, T. Roser, and J. A. Stewart, G. R. Court Phys. Rev. Lett. 64, 2627 (1990).

[6] St. Goertz, et al. Nucl. Instr. E Meth. A356:20 (1995).

[7] C.D. Jeffries, Phys. Rev. 106 (1957) 164.

[8] C.D. Jeffries, Phys. Rev. 117 (1960) 1056.

[9] M. Borghini, J. Phys. Lett. 26A, 242 (1968).

[10] D.G. Crabb and W. Meyer Annu. Rev. Nucl. Part. Sci. 47:67-109 (1997).

[11] M. Goldman, J. Magn. Reson. 17:393 (1975).

[12] G.R. Court, D.W. Gifford, P. Harrison, W.G. Heyes and M.A. Houlden., Nucl. Instr. and Meth. A 324:433 (1993).

[13] W. Tobias Measurements of the Proton and Deuteron Spin Structure Functions $g_{1}$ and $g_{2}$. Ph.D. thesis, University of Virginia (2001).

[14] P. Mckee. The Spin Structure Function of the Proton From SLAC Experiment E155. Ph.D. thesis, University of Virginia, Charlottesville (2000).

[15] D.C. Crabb, D. Day, et al. Nucl. Instr. 83 Meth. A356:9 (1995).

[16] D.G. Crabb International Workship: Polarized Sources and Targets, eds. A. Gute, S. Lorenz E. Steffens. University of Erlangen-Nürnberg. 340 (1999).

[17] D.G. Crabb Proc. 9th Int. Symp. High-Energy SPin Phys., Vol. 2:289, eds. W Meyer, E Steffens Theil. Germany: Springer Verlag (1991). 
[18] K.H. Althoff, et al. Proc. 4th Wkshp Polar. Target Mat. Tech., ed. W Meyer, p.23. Bonn: University of Bonn (1984).

[19] W. Nelson, H. Hirayama and D. Rogers, The EGS4 Code System, Slac-Report265, Stanford Linear Accelerator Center, (1985).

[20] P. Hautle Scintillating Polarized Targets for Spin Physics: Progress and Prospects, Talk given July 3-6 2002.

[21] P.E. Bosted, D.G. Crabb and K. Griffeon et al., A Proposal to Measure the $A$-Dependence of $J / \psi$ and $\psi$ 'Photoproduction, SLAC-Proposal E160 (2000).

[22] P.E. Bosted, D.G. Crabb et. al., Proposal to Measure $\Delta \sigma^{\gamma N}(k)$ and the High Energy Contribution to the Gerasimov-Drell-Hearn Sum Rule, SLAC-Proposal E159 (2000).

[23] P.E. Bosted, D.G. Crabb et. al., Proposal to Measure the Gluon Spin Distribution Using Polarized Open Charm Photoproduction, SLAC-Proposal E161 (2000).

[24] L.D. Landau and I.Ya. Pomeranchuk., Dokl. Akad. Nauk SSSR 92 (1953), p. 535 735 . 


\section{Appendix A}

\section{Instructions for running EGS4}

To run an EGS simulation there are two main programs that you need to run: pegs and xyzemf.

PEGS4 is a program used to generate a scattering parameter data file for each material in the sample in desired energy range. Once it is run for each material the output data files, labeled PEGS4. DAT need to be combined into a single pegs4. dat file (note the lower case).

When EGS4 runs, it reads the pegs4. dat file to find data for the media listed in the input.inp file. It assumes that the data for all the media contained in the pegs4. dat file were run using the same kinematics, energy limits etc.

The user is able to specify a material by inputting the material's density, the number of different kinds of atoms, and for each different kind of atom its atomic number, its weight and its proportion proportion by number or weight.

When inputting a new material the user has three different options: ELEMENT, COMPOUND and MIXTURE. The ELEMENT option is used if the material only has one kind of atom. In this instance only the atomic symbol is necessary. The 
COMPOUND option can be used when there is more than one different kind of atom and the proportions by number of atoms is known. The MIXTURE option is similar to the COMPOUND option, but is used when the atomic proportions are given by number instead of weight. With any of the three material types the default values can be overwritten by the user for isotopes, non-standard densities etc.

For materials composed of multiple atom types used in the simulations discussed earlier, such as ammonia or LiD beads in liquid He, the MIXTURE option was used. For other elemental materials, like aluminum or argon, use the ELEMENT option.

xyzemf is the "main" EGS4 program that contains all the main physics. It was modified from the original xyzdos program to simulate the presence of the magnetic fields in the $\hat{x}, \hat{y}$ and $\hat{z}$ directions. xyzdos is a simplified user code that one can use to run the main egs code. It was originally written in MORTRAN, but can be converted to FORTRAN for easier editing. It allows the user to define volumes in cartesian coordinates or to approximate other coordinates by using smaller voxel sizes.

Compiling the xyzemf code can be done using the following command:

g77 -c -0 -fcase-lower -fno-underscoring -malign-double

-fno-automatic -finit-local-zero XYZEMF.f

and then it can be linked using the command:

g77 -o XYZEMF XYZEMF. o

The latest version of xyzemf is xyzemf 6 .

To simplify the input of multiple materials at different energies a perl script called spectrum.pl was written. It allows the user to either increment incident particle 
energies manually or through a pre-defined list.

The script takes the incident energy, modifies the PEGS4. INP file, runs pegs4 for each material at the desired energy, then runs xyzemf itself after modifying the input.inp file to the correct energies. It then searches the output of xyzemf, a file called output.1st, to find the dose deposited per particle in each voxel.

To run EGS4 the user must first create a new PEGS4. INP files for each new material. An example pegs4.inp file is given in Appendix D. Page 69 of the egs4 user manual has a list of all the elements that pegs uses. Next, the user must change the material variable at the top of spectrum.pl. These variables are labeled material_o, material_1 etc. If the user wants to use a single energy for a number of trials, or do calculations on a wide spectrum of energies they can modify the energy array at the top of spectrum.pl entitled energy_list.

The next step is to modify the input.inp file, which contains most of the input variables. An example of such an input file is located in Appendix $\mathrm{C}$ with a detailed listing of what each record means in Appendix B.

Once those files have been modified the spectrum.pl file can be executed. The script asks for several input parameters and for labels so that the output directories can be easily identifiable. The script the runs PEGS and EGS for each energy. The output is printed to the screen and saved in Output.1st. The perl script then scans the Output.lst file for the dose deposited (in arbitrary units) in each voxel. It then writes a number of output files that can be easily graphed in gnuplot or other such graphing programs. The output files are labeled in the following format: \#_x_y_const, \#_z_const, energy_deposited. The \#_x_y_const files show the dose deposited for a constant $x=y$ as a function of $\mathrm{z}$. These files contain $\mathrm{z}$ in the first column and 
the dose deposited in arbitrary units in the second column. They allow the user to determine the dose as a function of z. The other files, named \#_z_const are similar, but allow the user to see the dose deposited for a constant $\mathrm{z}$ and a function of $\mathrm{x}$ and $\mathrm{y}$ in a 3-d plot. The format of these files is $\mathrm{x}$ in the first column, $\mathrm{y}$ in the second and the dose deposited in the third. Gnuplot can be used to fit the data to polynomials. Typically, a 7 th or 8 th order polynomial fits the curves very well. The total energy deposited can then be used to calibrate the dose deposited to get the amount of energy deposited as the shower progresses through the material. 


\section{Appendix B}

\section{Record information for input.inp}

Record 1 TITLE:

Can be up to 80 characters.

Record 2 NMED:

The number of media - defaults to 1 .

Record 3 (NMED times)

Media names, left justified. Note that entire volume is initially set to medium 1.

Record 4 ECUT,PCUT,ESTEPE(1 TO NMED):

Electron (total) and photon global cutoff energies in MeV ESTEPE for each medium in the problem.

Record 5 IMAX, JMAX, KMAX:

number of voxels in the $\mathrm{X}, \mathrm{Y}, \mathrm{Z}$ directions - if $<0$, it means that number of equally spaced boundaries will be input.

Record 6 et seq, repeated for $\mathrm{X}, \mathrm{Y}$ and $\mathrm{Z}$ directions separately i.e. repeat the following replacing ( $\mathrm{I}$ and $\mathrm{X}$ ) by $(\mathrm{J}$ and $\mathrm{Y}$ ) and ( $\mathrm{K}$ and $\mathrm{Z}$ ) respectively.

There is a maximum of 22 voxels in each direction.

if IMAX $>0$ then input, one per line, the IMAX $+1 \mathrm{X}$ boundaries 
if IMAX $<0$ input smallest $\mathrm{X}$ boundary, followed by ABS(IMAX) pairs one pr/line: voxel width, number of voxels with this width

for example: starting at Record 5

$$
\begin{aligned}
& -1,-1,-1 \\
& 0.0 \\
& 1.0,16 \\
& 0.0 \\
& 1.0,16 \\
& 0.0 \\
& 1.0,16
\end{aligned}
$$

defines a $16 \times 16 \times 16$ cube of $1 \mathrm{~cm}^{3}$ voxels with a total of 4097 regions. or

$$
\begin{aligned}
& -1,-1,3 \\
& 0.0 \\
& 1.0,16 \\
& 0.0 \\
& 1.0,16 \\
& 0.0 \\
& 5.0 \\
& 10.0
\end{aligned}
$$

defines a 16x16x10 cube with $1 \times 1 \times 5 \mathrm{~cm}$ voxels stacked 2 deep

Record 7 et seg IL,IU, JL,JU, KL,KU, MEDIUM, DENSITY:

Line is repeated until a blank line is found. All regions default to medium 1 with its default density unless changed here. For all voxels with:

$$
\begin{aligned}
& \mathrm{IL}<=\mathrm{I}<=\mathrm{IU} \\
& \mathrm{JL}<=\mathrm{J}<=\mathrm{JU} \\
& \mathrm{KL}<=\mathrm{K}<=\mathrm{KU}
\end{aligned}
$$

the medium used is MEDIUM and the density used is DENSITY. If DENSITY $=0.0$, the default value for that medium is used (faster than entering default density here) If IU and IL are non-zero, the rest default to all J,K.

Record 8 et seq IL,IU, JL,JU, KL,KU,IZSCAN:

Just like above except these are the regions for which the dose will be output. IZSCAN non-zero to get z-scan per page, otherwise output is an x-scan per page. 


\section{Record 9 XLOWER, XUPPER:}

Boundaries of beam in $\mathrm{X}$ direction, in $\mathrm{cm}$-if XLOWER is zero, a value near middle is taken If XUPPER is zero, no extent in $\mathrm{X}$ direction

\section{Record 10 YLOWER,YUPPER:}

as for $\mathrm{X}$ direction

\section{Record 11 THETAZ,THETAX,THETAY:}

THETAZ: Angle of beam to $\mathrm{Z}$ axis (0 is normal) in degrees if THETAZ is zero, others assumed normal(i.e.90 deg) If THETAZ is non-zero - and others both are zero, THETAX is as large as possible - i.e. max cos allowed, and THETAY is $90 \mathrm{deg}$ - if THETAX is non-zero, it may be reduced if too large, and THETAY will be choosen to normalize the direction cosines note: see NRCC code INHOM for examples of more complex incident beams

Record 12 EIN, IQIN, NCASE, IWATCH, TIMMAX, INSEED:

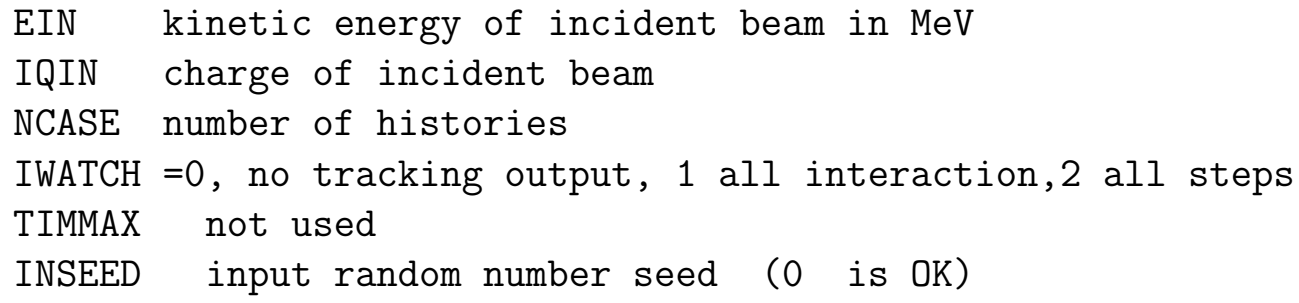

Record $13 \mathrm{~B}_{x}, \mathrm{~B}_{y}, \mathrm{~B}_{z}$ :

In units of the inverse curvature of the magnetic field:

$\frac{1}{r}=\frac{q v B}{m v^{2}}$ with $v=c$ 


\section{Appendix $\mathrm{C}$}

\section{input.inp}

Dbut Irradiation with $19.0 \mathrm{MeV}$ photons at NIST Full volume 1

ARGON

$0.0010,0.0010,0.01,0.01$

$-1,-1,21$

0.0

$0.15625,16$

0.0

$0.15625,16$

0.0

0.71

1.26

1.81

2.36

2.91

3.46

4.01

4.56

5.11

5.66

6.21

6.76

7.31

7.86

8.41

8.96 


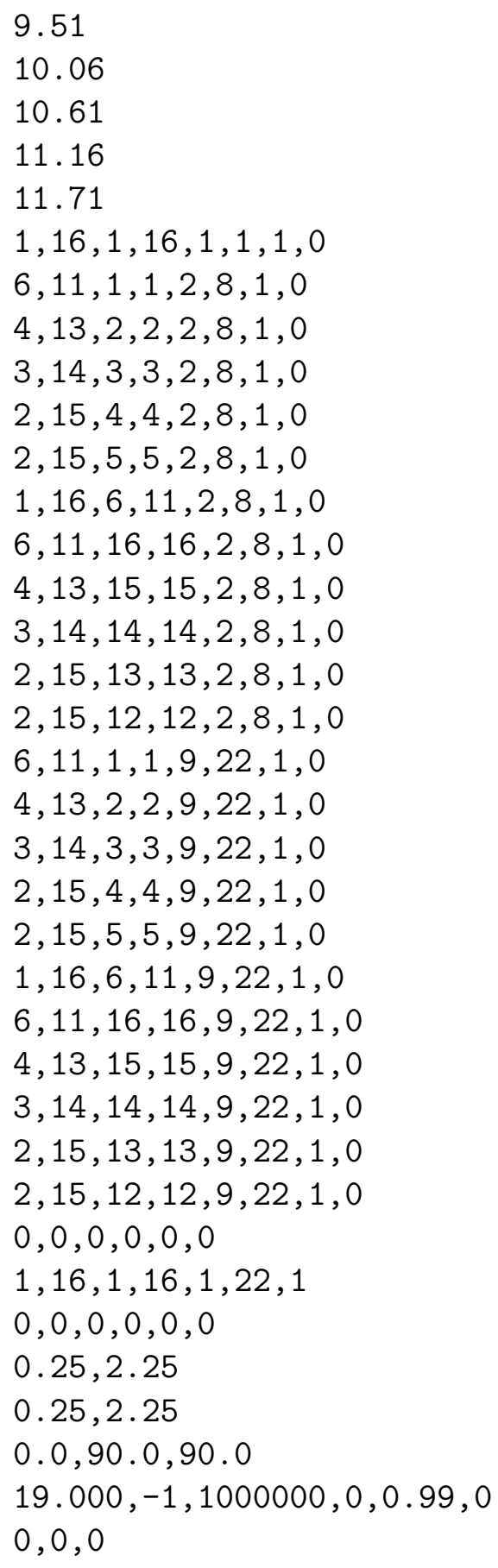




\section{Appendix D}

\section{pegs4.inp}

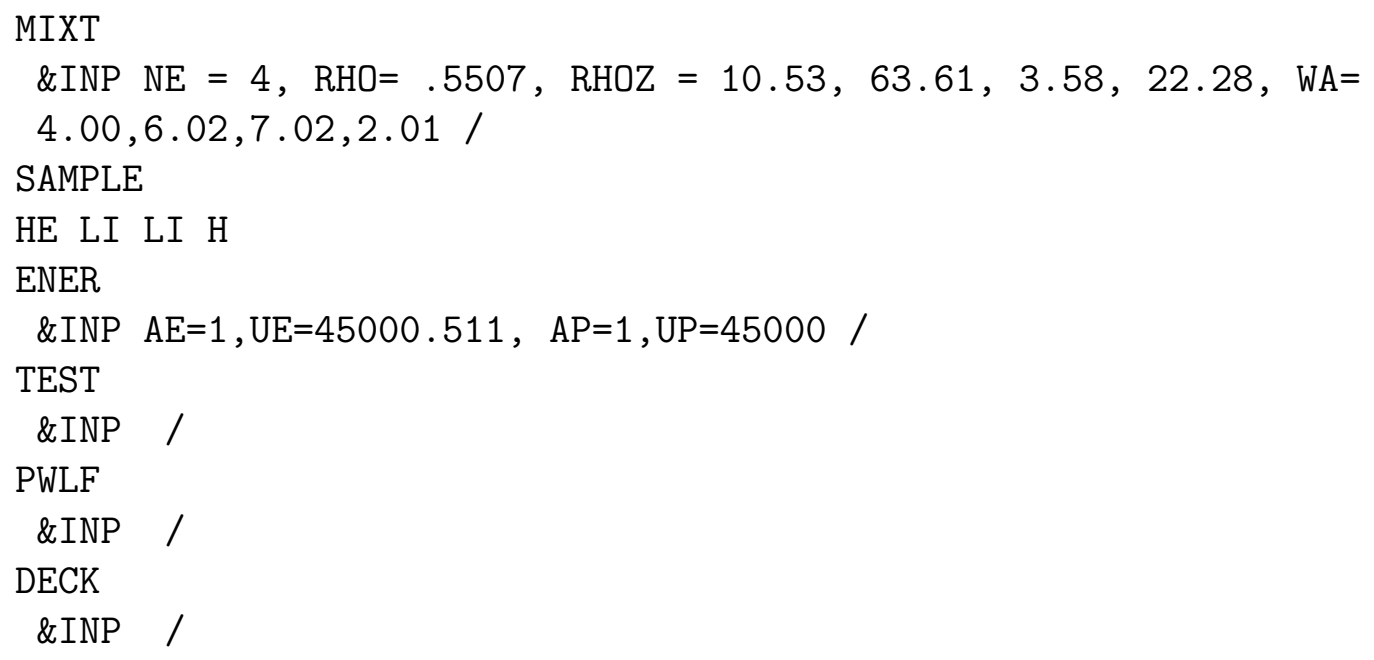




\section{Appendix E}

\section{Sample EGS4 Output}

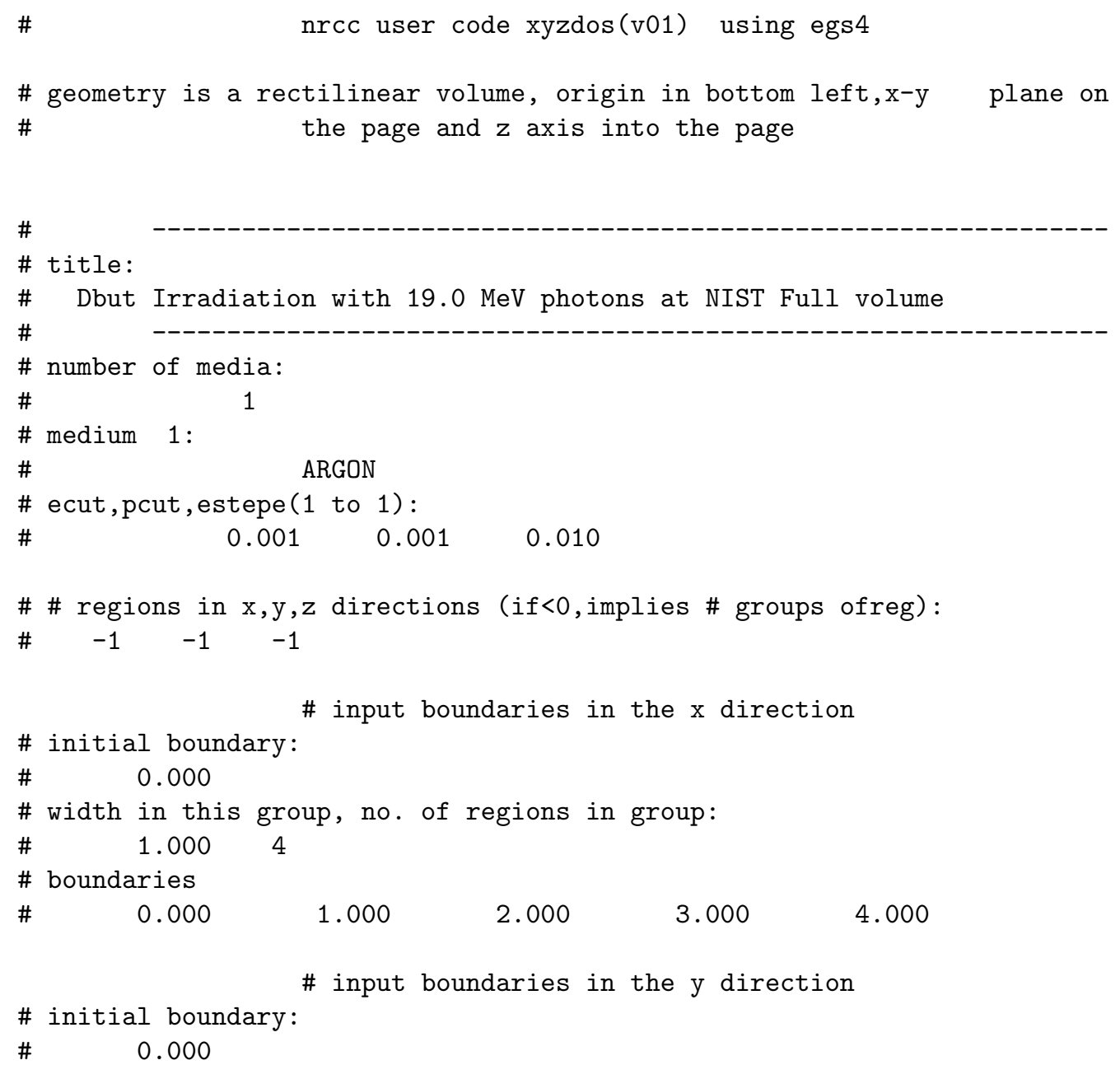




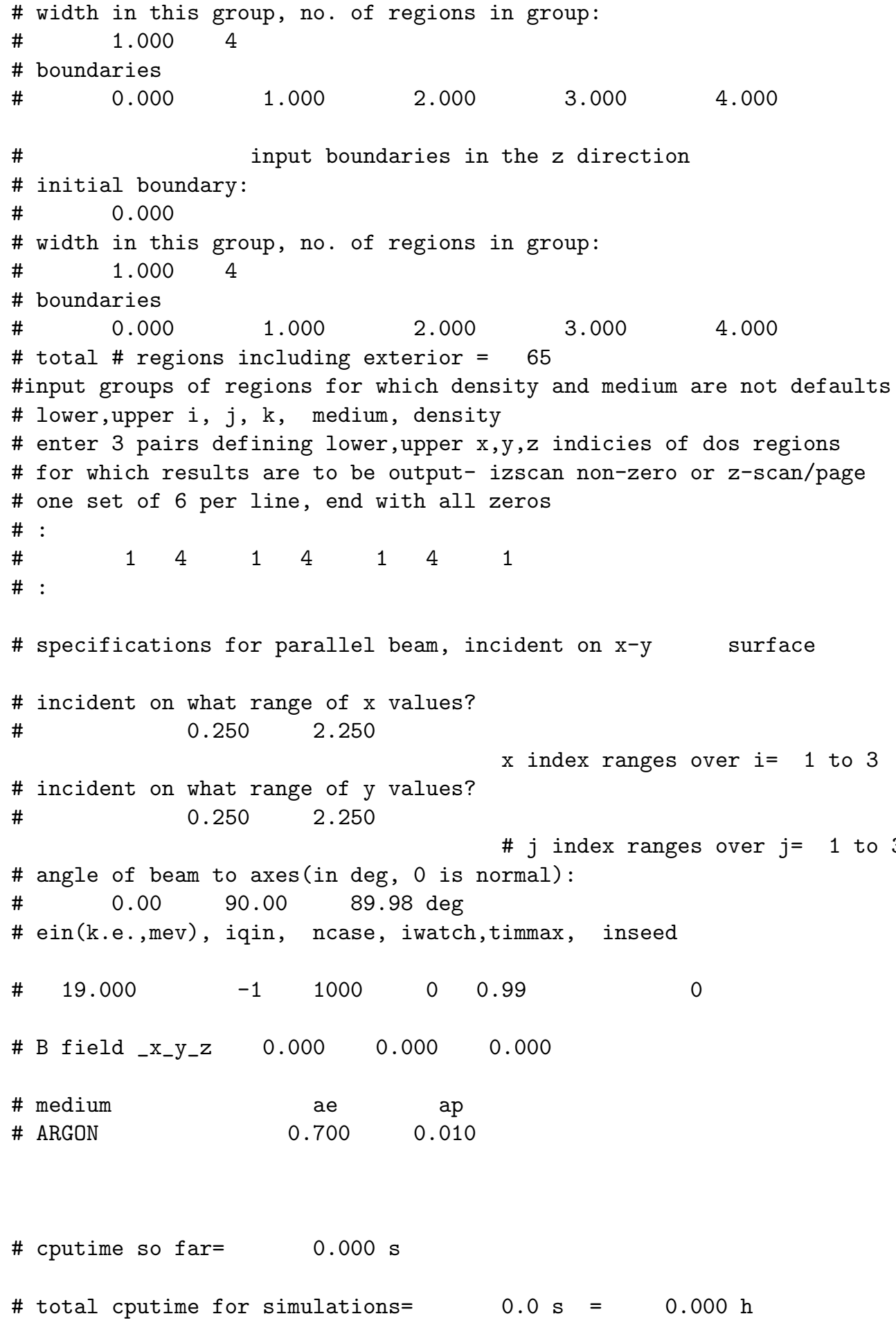


\# Dbut Irradiation with $19.0 \mathrm{MeV}$ photons at NIST Full volume \# xyz(v01) dose outputs Gy.*cm**2 (or Gy/incident particle for 0 area)

\begin{tabular}{|c|c|c|c|c|c|c|c|c|c|}
\hline \multirow{3}{*}{ \# ybounds } & \multicolumn{2}{|r|}{$\#$ for $x=$} & \multicolumn{2}{|c|}{0.000 to } & 1.000 & \multirow[t]{2}{*}{1} & \multirow[b]{2}{*}{3.000} & \multirow{2}{*}{\multicolumn{2}{|c|}{4.000}} \\
\hline & & .000 & 1.000 & & 2.000 & & & & \\
\hline & $j=$ & 1 & & 2 & & 3 & & 4 & \\
\hline zbounds & & $0.000)$ & & & & & & & \\
\hline 1.000 & 1 & $1.479 \mathrm{E}-10$ & $7.6 \%$ & $1.809 \mathrm{E}-10$ & $5.7 \%$ & $4.308 \mathrm{E}-11$ & $13.7 \%$ & $8.662 \mathrm{E}-13$ & $99.9 \%$ \\
\hline 2.000 & 2 & $1.437 \mathrm{E}-10$ & $8.8 \%$ & $1.946 \mathrm{E}-10$ & $6.0 \%$ & $5.062 \mathrm{E}-11$ & $11.3 \%$ & $1.265 \mathrm{E}-12$ & $56.5 \%$ \\
\hline 3.000 & 3 & $1.295 \mathrm{E}-10$ & $8.4 \%$ & $1.664 \mathrm{E}-10$ & $9.8 \%$ & $5.803 E-11$ & $10.7 \%$ & $8.806 \mathrm{E}-12$ & $39.2 \%$ \\
\hline 4.000 & 4 & $9.026 \mathrm{E}-11$ & $11.6 \%$ & $1.162 \mathrm{E}-10$ & $8.6 \%$ & $5.684 \mathrm{E}-11$ & $12.8 \%$ & $1.596 \mathrm{E}-11$ & $17.8 \%$ \\
\hline
\end{tabular}

\begin{tabular}{|c|c|c|c|c|c|c|c|c|c|}
\hline \multirow{2}{*}{$\begin{array}{r}\# \text { ybounds: } \\
\text { \# }\end{array}$} & \multicolumn{2}{|r|}{$\#$ for $x=$} & \multicolumn{2}{|c|}{1.000 to } & $\begin{array}{c}2.000 \\
2.000\end{array}$ & 2 & \multirow[t]{2}{*}{3.000} & \multicolumn{2}{|r|}{4.000} \\
\hline & $j=$ & 1 & & 2 & & 3 & & 4 & \\
\hline zbounds & & $0.000)$ & & & & & & & \\
\hline 1.000 & 1 & $2.124 \mathrm{E}-10$ & $4.6 \%$ & $2.308 \mathrm{E}-10$ & $2.5 \%$ & $5.844 \mathrm{E}-11$ & $12.1 \%$ & $2.573 \mathrm{E}-13$ & $75.8 \%$ \\
\hline 2.000 & 2 & $2.264 \mathrm{E}-10$ & $6.1 \%$ & $2.424 \mathrm{E}-10$ & $4.9 \%$ & $7.045 \mathrm{E}-11$ & $9.5 \%$ & $2.572 \mathrm{E}-12$ & $57.2 \%$ \\
\hline 3.000 & 3 & $1.759 \mathrm{E}-10$ & $8.8 \%$ & $2.068 \mathrm{E}-10$ & $5.1 \%$ & $8.791 \mathrm{E}-11$ & $9.7 \%$ & $1.081 \mathrm{E}-11$ & $27.8 \%$ \\
\hline 4.000 & 4 & $1.170 \mathrm{E}-10$ & $7.4 \%$ & $1.323 \mathrm{E}-10$ & $6.1 \%$ & $9.179 \mathrm{E}-11$ & $10.6 \%$ & $2.316 \mathrm{E}-11$ & $22.8 \%$ \\
\hline
\end{tabular}

\begin{tabular}{|c|c|c|c|c|c|c|c|c|c|}
\hline \multirow{3}{*}{ \# ybounds } & \multicolumn{2}{|r|}{$\#$ for $x=$} & \multicolumn{2}{|c|}{2.000 to } & 3.000 & \multirow[t]{2}{*}{3} & \multirow[b]{2}{*}{3.000} & \multirow{2}{*}{\multicolumn{2}{|c|}{4.000}} \\
\hline & & .000 & 1.000 & & 2.000 & & & & \\
\hline & $j=$ & 1 & & 2 & & 3 & & 4 & \\
\hline zbounds & ( & $0.000)$ & & & & & & & \\
\hline 1.000 & 1 & $4.614 \mathrm{E}-11$ & $18.2 \%$ & $6.600 \mathrm{E}-11$ & $15.4 \%$ & 1. $297 \mathrm{E}-11$ & $19.3 \%$ & $0.000 \mathrm{E}+00$ & $0.0 \%$ \\
\hline 2.000 & 2 & $5.655 \mathrm{E}-11$ & $10.9 \%$ & $7.085 \mathrm{E}-11$ & $16.6 \%$ & $1.288 \mathrm{E}-11$ & $19.3 \%$ & $2.797 \mathrm{E}-12$ & $61.0 \%$ \\
\hline 3.000 & 3 & $7.632 \mathrm{E}-11$ & $8.3 \%$ & $8.389 \mathrm{E}-11$ & $10.9 \%$ & $2.385 \mathrm{E}-11$ & $12.7 \%$ & $2.766 \mathrm{E}-12$ & $45.8 \%$ \\
\hline 4.000 & 4 & $7.010 \mathrm{E}-11$ & $13.3 \%$ & $7.109 \mathrm{E}-11$ & $11.0 \%$ & $3.359 \mathrm{E}-11$ & $17.1 \%$ & $1.529 \mathrm{E}-11$ & $22.9 \%$ \\
\hline
\end{tabular}

\begin{tabular}{|c|c|c|c|c|c|c|c|c|c|}
\hline \multirow{3}{*}{ \# ybounds } & \multicolumn{2}{|r|}{$\#$ for $x=$} & \multicolumn{2}{|c|}{3.000 to } & 4.000 & \multirow[t]{2}{*}{4} & \multirow[b]{2}{*}{3.000} & \multirow{2}{*}{\multicolumn{2}{|c|}{4.000}} \\
\hline & & 000 & 1.000 & & 2.000 & & & & \\
\hline & $j=$ & 1 & & 2 & & 3 & & 4 & \\
\hline zbounds & & $0.000)$ & & & & & & & \\
\hline 1.000 & 1 & $9.071 \mathrm{E}-16$ & $99.9 \%$ & $0.000 \mathrm{E}+00$ & $0.0 \%$ & $0.000 \mathrm{E}+00$ & $0.0 \%$ & $0.000 \mathrm{E}+00$ & $0.0 \%$ \\
\hline 2.000 & 2 & $2.074 \mathrm{E}-12$ & $51.1 \%$ & $1.703 \mathrm{E}-12$ & $99.9 \%$ & $4.890 \mathrm{E}-13$ & $99.9 \%$ & $0.000 \mathrm{E}+00$ & $0.0 \%$ \\
\hline 3.000 & 3 & $3.205 \mathrm{E}-12$ & $38.4 \%$ & $5.323 \mathrm{E}-12$ & $30.9 \%$ & $3.228 \mathrm{E}-12$ & $54.3 \%$ & $3.039 \mathrm{E}-14$ & $99.9 \%$ \\
\hline 4.000 & 4 & $1.026 \mathrm{E}-11$ & $17.8 \%$ & $2.434 \mathrm{E}-11$ & $15.3 \%$ & $1.487 \mathrm{E}-11$ & $32.1 \%$ & $3.121 \mathrm{E}-12$ & $47.6 \%$ \\
\hline
\end{tabular}

